\title{
Compressed Sensing-Aided Multi-Dimensional Index Modulation
}

\author{
Siyao Lu, Student Member, IEEE, Ibrahim A. Hemadeh, Member, IEEE, \\ Mohammed El-Hajjar, Senior Member, IEEE and Lajos Hanzo, Fellow, IEEE
}

\begin{abstract}
In this paper, we conceive a Compressed Sensing (CS) aided multi-dimensional Index Modulation (IM) scheme, where the benefits of Space-Time Shift Keying (STSK), Orthogonal Frequency Division Multiplexing (OFDM) relying on Frequency Domain (FD) IM and Spatial Modulation (SM) are explored. Explicitly, extra information bits are transmitted through the active indices of both the TAs and subcarriers, while striking a flexible design trade-off between the throughput and the diversity order. Furthermore, Compressed Sensing (CS) is invoked in both the transmitter and the receiver of our multi-dimensional system for the sake of improving the system's design flexibility, whilst reducing the detector's complexity. We first present the Maximum LikeLihood (ML) detector of the proposed CS-aided multi-dimensional IM system for characterizing the best-case bound of the proposed system's performance. Specifically, an upper bound is derived for the Average Bit Error Probability (ABEP) and it is observed that the derived theoretical upper bound becomes very tight with the ML detector simulation curves as the Signal-to-Noise Ratio (SNR) increases. Then we propose a reduced-complexity detector imposing only a modest Bit Error Ratio (BER) degradation, where we analyse the computational complexities of both ML detector and reduced-complexity detector. Furthermore, a Soft-Input Soft-Output (SISO) decoder is proposed for attaining a near-capacity performance, which is analyzed with the aid of Extrinsic Information Transfer (EXIT) charts. The maximum achievable rate of the proposed CS-aided multi-dimensional IM system relying both on ML detection and on our reduced-complexity based detector is also evaluated using EXIT charts. Additionally, the Discrete-Input Continious-Output Memoryless Channel (DCMC) capacity of the proposed CS-aided multi-dimensional IM scheme is formulated.
\end{abstract}

Index Terms-Space-Time Shift Keying (STSK), MultiDimenstional Index Modulation, Compressed Sensing (CS), Reduced-Complexity Detector, Soft Detection, EXIT Charts.

\section{INTRODUCTION}

$\mathbf{I}$ NDEX Modulation (IM) [1] [2] has attracted much attention as an emerging modulation concept as a benefit of its good performance, high data rate and hardware simplicity. Space-Time Shift Keying (STSK) [3] [4] was proposed as an advantageous multi-functional Multiple-Input MultipleOutput (MIMO) technique, which extends the concept of pure Spatial Domain (SD) Transmit Antenna (TA) activation of

L. Hanzo would like to acknowledge the financial support of the EPSRC projects EP/Noo4558/1, EP/PO34284/1, COALESCE, of the Royal Society's Global Challenges Research Fund Grant as well as of the European Research Council's Advanced Fellow Grant QuantCom.

The authors are with the School of Electronics and Computer Science, University of Southampton, Southampton SO17 1BJ, U.K. (e-mail:s118e14@ecs.soton.ac.uk; ibrahimhemadeh@gmail.com; meh@ecs.soton.ac.uk; lh@ecs.soton.ac.uk).

The research data supporting the paper can be obtained from the University of Southampton institutional repository: 10.5258/SOTON/D0820 the conventional Spatial Modulation (SM) [5] and Space-Shift Keying (SSK) [6] schemes to both the spatial and temporal dimensions. Hence STSK provides substantial transmit-diversity and multiplexing gains, whilst SM and SSK are only capable of attaining receive diverity gain. More specifically, STSK is based on the activation of $\mathcal{Q}$ appropriately indexed Space-Time (ST) Dispersion Matrices (DMs) within each STSK block duration, rather than at each classic symbol duration [4].

However, the majority of STSK studies only considered narrowband propagation scenarios instead of realistic wideband fading channels [4]. In order to mitigate the resultant linear distortion in wideband channels, typically Multi-Carrier (MC) modulation [7] is utilised, where Orthogonal Frequency Division Multiplexing (OFDM) is the most popular MC technique due to its robustness to dispersive wideband scenarios and its low-complexity implementation. Against this background, OFDM-STSK was conceived in [7] [8] for overcoming the performance degradation of STSK based transmissions over dispersive wideband channels. Recently, the concept of MultiSet Space-Time Shift Keying (MS-STSK) which is the extention of OFDM-STSK scheme was proposed in [9], where extra information is conveyed implicitly over the index of the activated combination of multiple TAs selected from a higher number of TA elements for the sake of achieving high data rates, whilst enhancing the system's performance.

More specifically, the IM transmitter maps additional information bits to the indices of TAs [10], subcarriers [11] or Time Slots (TSs) [12]. OFDM based IM (OFDM-IM) [13] [14] is a novel MC transmission technique, which is capable of enhancing the power efficiency and has been proposed as an alternative to classical OFDM. In OFDM-IM systems, part of the information is mapped to the Quadrature Amplituded Modulation (QAM) symbols, whilst the rest of the information is conveyed by the indices of the active subcarriers [11] in the Frequency Domain (FD). It has been shown in [2] that OFDMIM has the potential of improving the Bit Error Ratio (BER) of classical OFDM over frequency-selective fading channels at low Signal-to-Noise Ratios (SNRs). As a further development, OFDM-IM-MIMO techniques have been proposed in [15] [16], while providing a beneficial tradeoff between error performance and spectral efficiency. Additionally, in order to reduce the prohibitive computational complexity of the Maximum LikeLihood (ML) detector, several low complexity detectors have been proposed for the detection of OFDMIM-MIMO. Recently, IM techniques have been explored in different dimensions [10] [17], where it is shown that for a given transmission rate, improved performance is achieved by 
increasing the number of indexed dimensions. Specifically, in [10] a Generalized Space-Frequency IM (GSFIM) scheme was proposed for achieving increased rates as well as an improved performance compared to the conventional OFDM-MIMO scheme, where the information bits were encoded through indexing both into active TAs and subcarriers,. However, it becomes much more challenging to detect the active TAs, the active subcarrier indices and modulated symbols due to the strong interference imposed both on the OFDM-IM subblock and TAs. Although the ML detector is capable of achieving optimal performance, its prohibitive computational complexity makes it impractical for the GSFIM scheme. Hence, it is essential to conceive a low-complexity detection.

Compressed Sensing (CS) [18] is an attractive paradigm of casting signals represented in high-dimensional sparselypopulated spaces into signals that lie in spaces of significantly smaller dimensions. In recent years, due to the sparsity characteristic of IM related techniques, CS has been invoked for detecting sparse signals with a low-complexity. More specifically, low-complexity CS based detectors have been developed for exploiting sparsity of Generalized Spatial Modulation (GSM) symbols in [19] [20], where the single dimensional IM was applied in these literatures. Furthermore, [21] proposed both a CS assisted signalling strategy at the transmitter and a CS based low-complexity detector at the receiver, while achieving a higher spectral efficiency and providing an attractive detection performance at a low complexity. Recently, in [22], we proposed a CS-aided OFDMSTSK scheme relying on subcarrier IM, which combineed the benefits of STSK and OFDM-IM for striking a flexible tradeoff between the throughput and bit-error performance. More specifically, in order to further improve the tradeoff, as well as to decrease the complexity of the detector, CS was explored in both the transmitter and the receiver. Furthermore, the CS-aided OFDM-STSK-IM soft decoder by applying the ML detection was proposed for achieving a near-capacity performance. Therefore, in this paper we aim to extend our previous literature to the SD for attaining an improved spectral efficiency and to design a soft decoder using the proposed reduced-complexity detector for achieving a near-capacity performance.

Additionally, in order for any communications system to attain a low BER at SNRs close to the channel capacity [23], it is essential to invoke channel coding and iterative decoding. Hence numerous channel coding techniques have been proposed since the 1950s, including Hamming codes [24], Convolutional Codes (CC) [25], turbo codes [26] as well as diverse other channel coding techniques [27]. More specifically, Extrinsic Information Transfer (EXIT) charts [28] [29] were proposed as a powerful semi-analytical tools devised for analysing the convergence behaviour of iteratively decoded systems as well as for evaluating the maximum achievable throughput of a system.

Against the above background, the contributions of this paper are summarized as follows:

- We propose a CS-aided multi-dimensional IM system relying on both FD and SD IM for improving the performance by attaining an improved diversity gain, whilst increasing the system capacity by indexing multiple transmission entities compared both to the classical OFDMMIMO system and to our previously proposed CS-aided subcarrier IM system [22]. More explicitly, compared to the classical OFDM-STSK system and to our previously proposed CS-aided subcarrier IM system [22] relying on ML detection, the proposed system exhibits SNR-gains of about $8.75 \mathrm{~dB}$ and $4.15 \mathrm{~dB}$ at the BER of $10^{-5}$, respectively. Furthermore, an upper bound of the Average Bit Error Probability (ABEP) of the proposed CS-aided multi-dimentional IM system has also been derived and confirmed by computer simulations. The proposed design is a flexible multifunctional design that allows striking a flexible trade-off between performance, throughput and complexity, where the different components in the design can be configured in order to attain specific design requirements.

- A reduced-complexity so-called Simultaneous Matching Pursuit (S-MP) detector is proposed for simultaneously detecting Multiple Measurement Vectors (MMV) by exploiting the powerful CS principles, whilst mitigating the excessive complexity of the ML detector at the cost of a modest performance loss. Explicitly, the proposed S-MP detector is specifically designed for our proposed CSaided multi-dimentional IM system, where the active TAs and active subcarriers are first jointly detected and then they are utilised for reconstructing the ST symbols.

- Then the CS-aided multi-dimensional IM Soft-Input SoftOutput (SISO) decoders using both the ML detector and the proposed S-MP detector are conceived for iteratively exchanging extrinsic soft information between the constituent decoders in order to achieve a near-capacity performance. More specifically, EXIT charts are used for visually characterising the flow of soft-information between the constituent decoders of our concatenated structure. We also construct EXIT charts for evaluating the maximum achievable rate of the proposed system using both the ML detector and the S-MP detector. Furthermore, the Discrete-Input Continious-Output Memoryless Channel (DCMC) capacity of the proposed CSaided multi-dimensional IM scheme is formulated and compared to the maximum achievable rate of the system derived from the EXIT charts.

The rest of the paper is organized as follows. In Section II, the system model of CS-aided multi-dimensional IM relying both on FD and SD IM is introduced. Then in Section III, the hard-decision based ML detector and the reduced-complexity detector are introduced. Additionally, soft-decision detection is also conceived in Section III. Our simulation results, EXIT chart analysis and system capacity estimates are provided in Section IV. Finally, our conclusions are offered in Section VI.

Notations: Bold uppercase and lowercase letters represent matrices and vectors, respectively; $\lfloor\cdot\rfloor$ is used to denote the integer floor operation; $(\cdot)^{-1},(\cdot)^{T},(\cdot)^{H}$ and $(\cdot)^{\dagger}$ represent the inverse operation, the transpose operation, the Hermitian transpose operation and the Moore-Penrose pseudoinverse operation, respectively; $\operatorname{diag}\{\cdot\}$ is used to denote the diagonal 
structure of a matrix; $\|\cdot\|$ denotes the Frobenius norm; $\mathbb{C}^{r \times c}$ illustrates the size of a complex-valued matrix, where the size is $(r \times c) ; L(\cdot)$ indicates Log Likelihood Ratios (LLRs) of a bit sequence.

\section{SySTEM MODEL}

In this section, we introduce the transceiver architecture of the proposed CS-aided multi-dimensional IM system relying on $N_{t}$ TAs and $N_{r}$ Receive Antennas (RAs), which is illustrated in Fig. 1. In Fig. 1, we consider OFDM having $N_{c}$ subcarriers, which are then equally divided into $G$ subcarrier groups and each subcarrier group contains $N_{f}=N_{c} / G$ subcarriers in the FD. Additionally, the Virtual Domain ${ }^{1}$ (VD) is applied for the proposed system shown in Fig. 1. More explicitly, there are $N_{v}$ available subcarrier indices in the VD for each subcarrier group, where the dimension $N_{v}$ of the VD is higher than the dimension $N_{f}$ of the $\mathrm{FD}^{2}$ [18] [30]. Then, the measurement matrix $A$ shown in Fig. 1 compresses the $N_{v}$-dimensional VD symbols into the $N_{f}$-dimensional FD symbols for each subcarrier group using CS.

In this paper, we focus on the transmission and detection of a single group instead of $G$ groups, because each subcarrier group has the same procedures, as shown in Fig. 1. The transmitter model is introduced in Section II-A, followed by the receiver model in Section II-B.

\section{A. Transmitter}

The transmitter model of the proposed CS-aided multidimensional IM system is shown in Fig. 1. As seen in Fig. 1, the information bits of $b G$ are partitioned into $G$ groups and then each group of $b$ bits are split into smaller segments of $b_{1}, b_{2}$ and $b_{3}$ bits, where the first $b_{1}$ bits are mapped to the Antenna Selector, the remaining $b_{2}$ and $b_{3}$ bits are processed by the Subcarrier Index (SI) Selector and STSK Encoder, respectively.

1) Antenna Selector: The first incoming $b_{1}$ bits are processed by the Antenna Selector of Fig. 1, which selects $M$ TAs out of the $N_{t}$ available TAs while obtaining $N_{A C}$ Antenna Combinations (ACs) in total ${ }^{3}$. Compared to the SM scheme, a specific combination of TAs out of $N_{A C}$ ACs is activated during each transmission block in the proposed system instead of just activating a single TA. Inspired by the concept of the Distinctive Antenna Combination (DAC) scheme proposed in [9], we assign $M$ unique TAs to each $\mathrm{AC}$ in order to avoid the correlation caused by sharing the same TA element among different ACs. Additionally, the specific AC selected for transmitting symbols in each subcarrier group depends on the $b_{1}=\left\lfloor\log _{2}\left(N_{A C}\right)\right\rfloor$ information bits controlling the Antenna Selector in Fig. 1. Specifically, a look-up table

\footnotetext{
${ }^{1}$ The virtual domain is the symbols domain before applying CS as shown in Fig. 1, the concept of which is firstly introduced in [21] to illustrate the application of CS technique at the transmitter for improving the spectral efficiency.

${ }^{2}$ The FD here represents the symbol domain after the CS process and this then forms the OFDM symbol that is transmitted as shown in Fig. 1.

${ }^{3}$ The proposed system applying Antenna Selector becomes the normal STSK scheme if we have $M=N_{t}$, and the proposed system can transmit more data bits by applying $M<N_{t}$ without affecting the performance.
}

TABLE I

A LoOK-Up TABle EXAMPLE For THE ANTENNA SElECtor With $M=2$ AND $N_{t}=4$

\begin{tabular}{|c|c|c|}
\hline$b_{1}$ & Indices & Allocation \\
\hline [0] & $\{1,2\}$ & {$\left[\begin{array}{llll}T A^{1} & T A^{2} & 0 & 0\end{array}\right]$} \\
\hline [1] & $\{3,4\}$ & {$\left[\begin{array}{llll}0 & 0 & T A^{3} & T A^{4}\end{array}\right]$} \\
\hline
\end{tabular}

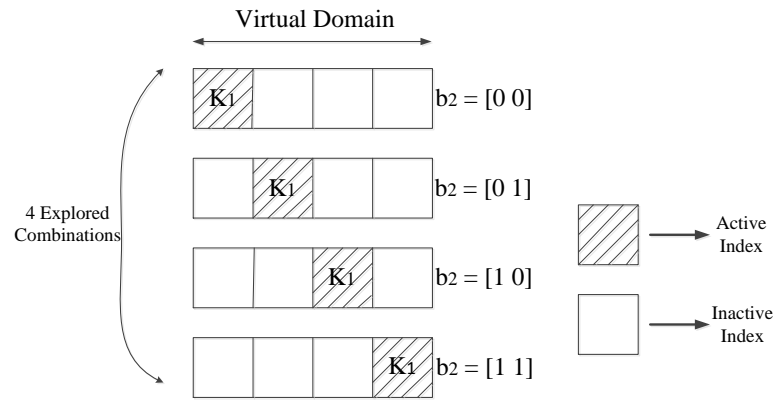

Fig. 2. The selection procedure of the SI Selector for $K=1$ and $N_{v}=4$.

example of the Antenna Selector is provided in Table I for $M=2, N_{t}=4$ and $N_{A C}=2$. In this example, when the first incoming bit is $b_{1}=[0]$, then the first and second TAs, namely $T A^{1}$ and $T A^{2}$ will be selected as unique $\mathrm{AC}$ for transmitting symbols in a specific subcarrier block, while the other two TAs remain inactive. Similarly, if the first incoming bit is $b_{1}=[1]$, then the third and fourth TAs denoted by $T A^{3}$ and $T A^{4}$ are activated for the transmission of symbols.

2) Subcarrier Index Selector: Then the second incoming sequence of $b_{2}$ bits is applied to the SI Selector in Fig. 1. For each subcarrier block, only $K$ active subcarriers are chosen out of the $N_{v}$ available subcarriers in the VD, and the specific selection procedure is determined by the information bits $b_{2}$. For example, assuming that the SI Selector is designed to select $K=1$ active subcarriers out of the $N_{v}=4$ available subcarriers in each subcarrier group, there are 4 possible SI combinations in total. Then, one out of $N_{S I}=4$ combinations is selected for each subcarrier group depending on the incoming bits $b_{2}$ and the corresponding selection procedure is illustrated in Fig. 2, where the shaded square represents the active subcarriers and the blank square indicates the inactive subcarriers. Specifically, in this example the first subcarrier is activated according to the incoming bits $b_{2}=[00]$, while the second, the third and the fourth subcarriers are selected for $b_{2}=[01], b_{2}=[10]$ and $b_{2}=[11]$, respectively. In each subcarrier group, the selected active subcarrier combination is fed into the Index Switch of Fig. 1 for allocating $K$ ST symbols generated from the STSK Encoder to the activated subcarriers.

3) STSK Encoder: According to [3], a total of $b_{3}=$ $K \log _{2}(Q L)$ bits are fed into the STSK Encoder to generate $K$ STSK codewords $\{\mathbf{X}[1], \ldots, \mathbf{X}[i], \ldots, \mathbf{X}[K]\}$ spreading the information over $M$ TAs over $T$ TSs in each subcarrier group, where each ST codeword $\mathbf{X}[i] \in \mathbb{C}^{M \times T}$ is generated by spreading an $L$-ary conventional constellation symbol over one out of $Q$ DMs. Additionally, the STSK Encoder is specified 


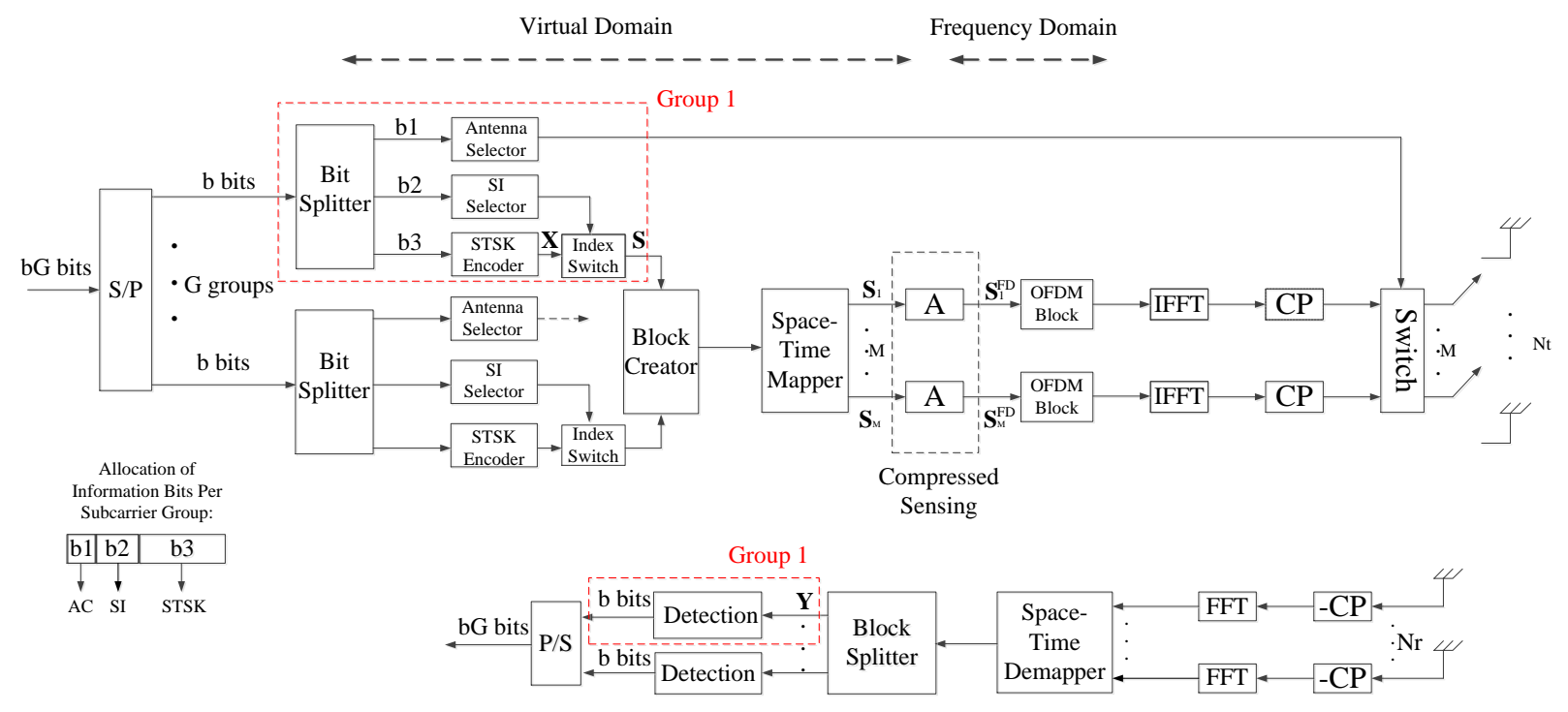

Fig. 1. The proposed multi-dimensional IM aided transceiver architecture.

by the five parameters $(M, N, T, Q, L)$, where $M, N$ and $T$ represent the number of TAs, RAs and TSs, respectively, while $Q$ and $L$ are the number of DMs and of $L$-ary constellation symbols. Note that we have $N=N_{r}$ in this paper. According to the specific SI combination of each subcarrier group gleaned from the Index Switch of Fig. 1, the $K$ STSK symbols ${ }^{4}$ generated by the STSK Encoder are mapped to the $K$ active subcarriers selected, while the inactive subcarriers are set to zero, which results in the symbol $\mathbf{S}$ in Fig. 1. Upon considering $b_{2}=[00]$ of the example shown in Fig. 2, the ST symbol generated after the active subcarrier allocation becomes $\mathbf{S}=\left\{\mathbf{X}\left[\begin{array}{llll}1] & \mathbf{0} & 0 & 0\end{array}\right\}\right.$.

4) Transmitter Model: Fig. 3 shows the ST structure of $\mathrm{S}$ per subcarrier group and illustrates the ST mapping process for the parameters of $N_{v}=4, N_{f}=2, N_{t}=4$ and $\operatorname{STSK}(2,2,2,2,2)$. Additionally, Fig. 3 portrays how the measurement matrix $A$ compresses the symbols of dimension $N_{v}=4$ observed in the VD into the symbols of dimension $N_{f}=2$ in the FD per subcarrier group. There are $G$ ST structures similar to $\mathbf{S}$, as illustrated in Fig. 3 for $G$ subcarrier groups. Then the Block Creator of Fig. 1 assembles these $G$ ST structures into a ST frame, which is then processed by the Space-Time Mapper of Fig. 1. Note that all processes in the following text only refer to a single subcarrier group instead of the entire frame, since all of them are processed identically. Specifically, the ST symbol S per subcarrier group is then mapped to $M$ TAs during $T$ TSs by the Space-Time Mapper of Fig. 1 and $M$ symbol sequences $\left\{\mathbf{s}_{1}, \ldots, \mathbf{s}_{M}\right\}$ are obtained at the $M$ TAs during a single TS, as shown in Fig. 1.

Observe in Fig. 1 and Fig. 3 that a measurement matrix

\footnotetext{
${ }^{4}$ Note that the number of STSK codewords generated by the STSK Encoder is the same as the number of active subcarriers in each subcarrier group for allocating $K$ STSK codewords to $K$ active subcarriers selected by the SI Selector of Fig. 1.
}

A of size $\left(N_{f} \times N_{v}\right)$ is applied to the ST mapped vectors for compressing the $N_{v}$-dimensional vectors $\mathbf{s}_{m}(m=$ $1,2, \ldots, M)$ of the VD into the $N_{f}$-dimensional vectors $\mathbf{s}_{m}^{F D}$ $(m=1,2, \ldots, M)$ of the FD, where $M$ OFDM symbols are constructed, while hosting $G$ groups of $N_{f}$-dimensional vectors. The vector $\mathbf{s}_{m}^{F D}$ at the output of the CS block of Fig. 1 is then mapped to the FD OFDM subcarriers, which can be written as

$$
\mathbf{s}_{m}^{F D}=\mathbf{A} \mathbf{s}_{m}
$$

In the transmitter of the proposed system shown in Fig. 1, the FD symbol vectors $\mathbf{s}_{m}^{F D}(m=1,2, \ldots, M)$ per group at $M$ TAs during each TS are transmitted over a specific active TAs combination out of $N_{A C}$ available combinations, as shown in Fig. 1 and Fig. 4 further illustrates the TA selection scheme with $M=2$ and $N_{t}=4$ as an example. More explicitly, as shown in Fig. 4, the FD symbol vectors $\mathbf{s}_{1}^{F D}$ and $\mathbf{s}_{2}^{F D}$ in each subcarrier group during a single TS are transmitted over the first and second activated TAs if the incoming data bit is $b_{1}=[0]$ and the other two TAs are inactive. The third and the fourth activated TAs are used for transmiting $\mathbf{s}_{1}^{F D}$ and $\mathbf{s}_{2}^{F D}$, if the incoming data bit is $b_{1}=[1]$.

\section{B. Receiver}

We consider a $\left(N_{r} \times N_{t}\right)$-MIMO system, where $N_{r}$ is the number of RAs as shown in Fig. 1. At the receiver side, the signals of the $G$ groups received from $N_{r}$ RAs during $T$ TSs are subjected to the Fast Fourier Transform (FFT) for detecting the FD output symbols. The channel matrix is $\mathbf{H}_{\alpha} \in \mathbb{C}^{N_{r} \times N_{t}}$ for $\alpha=1, \ldots, N_{f}$. As shown in Fig. 1, the Space-Time Demapper collects the $G$ groups FD symbols from $N_{r}$ RAs over $T$ TSs, and the ST demapped symbols are split into $G$ groups by the Block Splitter of Fig. 1. The symbols received by each subcarrier group are represented by 


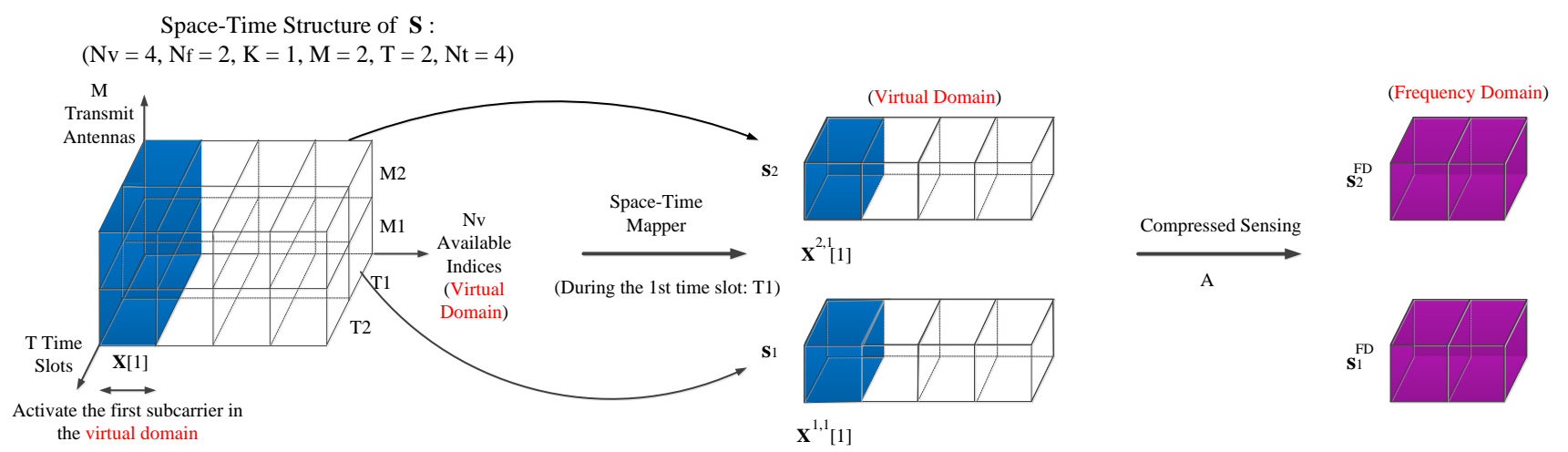

Fig. 3. Illustration of the structure for the frame symbol $\mathbf{S}$ per subcarrier group, the process of the Space-Time Mapper in the VD and the process of CS technique in the FD, while having $M=2 \mathrm{TAs}, T=2 \mathrm{TSs}, K=1$ active subcarrier out of $N_{v}=4$ available indices in the VD and $N_{f}=2$ subcarriers per subcarrier group in the FD. Additionally, the example given in Fig. 2 is applied and we have $b_{2}=[00]$.

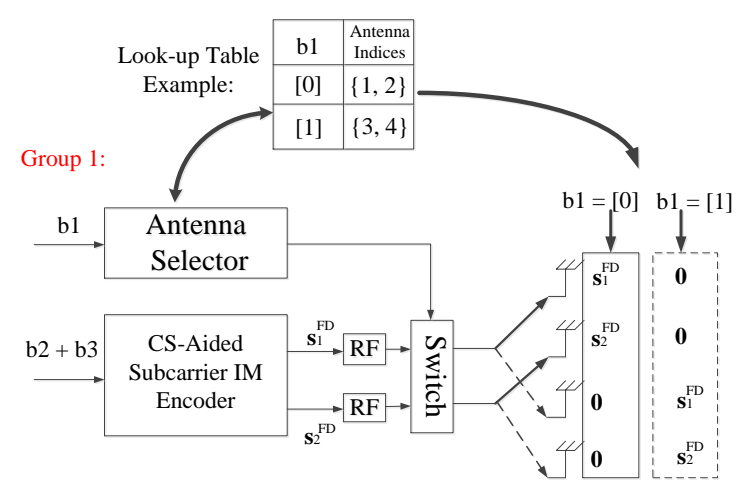

Fig. 4. The process of selecting active TAs with $M=2$ and $N_{t}=4$.

$\mathbf{Y}=\left\{\mathbf{Y}[1]^{T}, \ldots, \mathbf{Y}[\alpha]^{T}, \ldots, \mathbf{Y}\left[N_{f}\right]^{T}\right\}^{T}\left(\alpha=1, \ldots, N_{f}\right)$, as shown in Fig. 1, where $\mathbf{Y} \in \mathbb{C}^{N_{r} N_{f} \times T}$ and $\mathbf{Y}[\alpha] \in \mathbb{C}^{N_{r} \times T}$ characterize the ST structure and the ST symbol received at the $\alpha$-th subcarrier of each subcarrier group, respectively.

Then the signals $\mathbf{Y}[\alpha] \in \mathbb{C}^{N_{r} \times T}\left(\alpha=1, \ldots, N_{f}\right)$ received during $T$ TSs of each subcarrier group can be modelled as

$$
\mathbf{Y}[\alpha]=\mathbf{H}_{\alpha} \mathbf{I}_{A C} \mathbf{S}^{F D}[\alpha]+\mathbf{W}[\alpha],
$$

where the $\mathbf{S}^{F D}[\alpha] \in \mathbb{C}^{M \times T}$ denotes ST symbols at $N_{f}$ FD subcarriers transmitted from $M$ TAs over $T$ TSs, and $\mathbf{W}[\alpha] \in \mathbb{C}^{N_{r} \times T}$ represents the Additive White Gaussian Noise (AWGN) obeying the distribution of $\mathcal{C N}\left(0, \sigma_{n}^{2}\right)$ and $\sigma_{n}^{2}$ is the noise variance. Additionally, $\mathbf{I}_{A C} \in \mathbb{C}^{N_{t} \times M}$ in (2) denotes the $\left(N_{t} \times M\right)$-element submatrix, which explicitly describes the selection pattern of active TAs for each subcarrier group at the transmitter. More specifically, taking the example shown in Fig. 4 associated with $M=2$ and $N_{t}=4$, the $(4 \times 2)$ element submatrix $\mathbf{I}_{A C}$ activating the first and second TAs out of the 4 available TAs is generated, as shown in Fig. 5. Then after constructing the ST symbols for each subcarrier group by the Space-Time Demapper, the received signals are detected group-by-group as described in the following section.

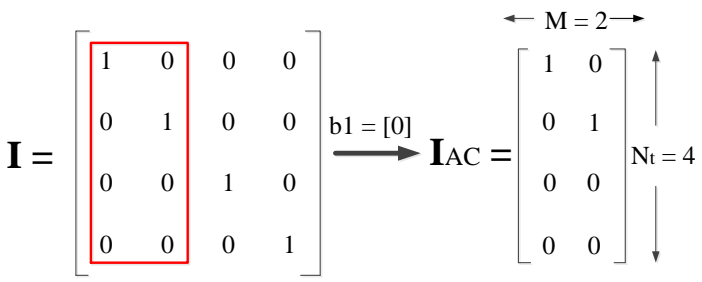

Fig. 5. The illustration of TA-selection submatrix, where it takes $M=2$ and $N_{t}=4$ as an example.

\section{Detection Techniques}

In the proposed CS-aided multi-dimensional IM system, the receiver has to detect the information bits conveyed by the STSK codewords at the active subcarriers plus the bits mapped to the active subcarrier indices, as well as the bits mapped to the active TAs, as shown in Fig. 1. Again, we consider both hard-decision ML detection and our reducedcomplexity Simultaneous Matching Pursuit (S-MP) detection, later followed by soft-decision detections.

As shown in Fig. 1, the received signal $\mathbf{Y}$ contains $N_{f}$ ST symbols at the $N_{f}$ subcarriers in the FD of each subcarrier group. According to the received signal model $\mathbf{Y}[\alpha] \in \mathbb{C}^{N_{r} \times T}$ $\left(\alpha=1, \ldots, N_{f}\right)$ shown in (2), we can rewrite $\mathbf{Y} \in \mathbb{C}^{N_{r} N_{f} \times T}$ as

$$
\mathbf{Y}=\mathbf{H} \overline{\mathbf{I}}_{A C} \mathbf{S}^{F D}+\mathbf{W},
$$

where $\mathbf{W} \in \mathbb{C}^{N_{r} N_{f} \times T}$ denotes the AWGN matrix of the $N_{f}$ subcarriers, and the channel matrix $\mathbf{H}$ associated with transmitting $N_{f}$ ST signals in each subcarrier group, which has a diagonal structure of size $\left(N_{r} N_{f} \times N_{t} N_{f}\right)$ and can be expanded as

$$
\mathbf{H}=\operatorname{diag}\left\{\mathbf{H}_{1}, \mathbf{H}_{2}, \ldots, \mathbf{H}_{N_{f}}\right\} .
$$

Similarly, $\overline{\mathbf{I}}_{A C} \in \mathbb{C}^{N_{t} N_{f} \times M N_{f}}$ denotes the TA selection pattern of $N_{f}$ subcarriers in each subcarrier group, which has 
the diagonal structure of

$$
\overline{\mathbf{I}}_{A C}=\operatorname{diag}\left\{\mathbf{I}_{A C}, \mathbf{I}_{A C}, \ldots, \mathbf{I}_{A C}\right\} .
$$

Additionally, $\mathbf{S}^{F D} \in \mathbb{C}^{M N_{f} \times T}$ represents the FD ST signals of the $N_{f}$ subcarriers transmitted from $M$ TAs over $T$ TSs, which are obtained after the SI modulation and CS, as shown in Fig. 1 and Fig. 3. In order to make the analysis more comprehensive, the transmitted ST signals $\mathbf{S}^{F D} \in \mathbb{C}^{M N_{f} \times T}$ in (3) are represented as

$$
\mathbf{S}^{F D}=\overline{\mathbf{A}} \mathbf{S}^{V D}=\overline{\mathbf{A}} \mathbf{I}_{S I} \mathbf{X},
$$

where $\overline{\mathbf{A}} \in \mathbb{C}^{M N_{f} \times M N_{v}}$ is the equivalent measurement matrix A shown in (1) invoked for compressing the $M$ virtual-domain vectors, and $\mathbf{S}^{V D} \in \mathbb{C}^{M N_{v} \times T}$ denotes the virtual-domain ST symbols, as shown in Fig. 1. Then $\mathbf{S}^{V D}$ is expanded as $\mathbf{S}^{V D}$ $=\mathbf{I}_{S I} \mathbf{X}$, where $\mathbf{X} \in \mathbb{C}^{M K \times T}$ represents $K$ STSK codewords generated by the STSK Encoder of Fig. 1. Additionally, the $K$ STSK codewords $\mathbf{X} \in \mathbb{C}^{M K \times T}$ are also illustrated in Fig. 3, where $K=1$ is considered and the shaded $\mathbf{X}[1]$ specifically illustrates the structure of $\mathbf{X} \in \mathbb{C}^{M K \times T}$. Additionally, $\mathbf{I}_{S I} \in$ $\mathbb{C}^{M N_{v} \times M K}$ illustrates the specific SI selection pattern of each subcarrier group while selecting $K$ active subcarriers out of $N_{v}$ available subcarriers in the VD. If we consider the example provided in Fig. 2 and Fig. 3 using $K=1, N_{v}=4$ and $M$ $=2$, then the $(8 \times 2)$-element submatrix $\mathbf{I}_{S I}$ for $b_{2}=\left[\begin{array}{ll}0 & 0] \text { is }\end{array}\right.$ written as

$$
\mathbf{I}_{S I}=\operatorname{diag}\left\{\left[\begin{array}{llll}
1 & 0 & 0 & 0
\end{array}\right]^{T},\left[\begin{array}{llll}
1 & 0 & 0 & 0
\end{array}\right]^{T}\right\},
$$

where the first index is activated out of the 4 available subcarrier indices in the VD.

According to (3) - (7), the received signal model for each subcarrier group can be finally expressed as

$$
\mathbf{Y}=\mathbf{H} \overline{\mathbf{I}}_{A C} \overline{\mathbf{A}} \mathbf{I}_{S I} \mathbf{X}+\mathbf{W}
$$

where $\overline{\mathbf{I}}_{A C}$ contains the TAs selection information and $\mathbf{I}_{S I}$ includes the SI selection information for the subcarrier group. Based on the received signal model of (8), hard-decision decoding is discussed in Section III-A, while soft-decision decoding is described in Section III-B.

\section{A. Hard-Decision Detection}

In this section, the hard-decision based ML detector and the reduced-complexity detector of the proposed CS-aided multidimensional system are proposed and an upper bound of the ABEP of the proposed system is also derived. The computational complexity of the two detectors is also compared. We assume perfect channel knowledge at the receiver.

1) Maximum Likelihood Detection: At the receiver, the ML detector makes a joint decision on the active TA indices, the active subcarrier indices and the constellation points at the corresponding active subcarrier indices by exploring all possible realizations of each subcarrier group. According to the system model in (8), $\overline{\mathbf{I}}_{A C}$ represents the specific realization of the active TA indices out of the $N_{A C}$ possible realizations in each subcarrier group and we use $\overline{\mathcal{I}}_{A C}(\gamma)\left(\gamma=1,2, \ldots, N_{A C}\right)$ to denote the realization of the active TA indices. Similarly, $\mathbf{I}_{S I}$ in (8) represents the specific realization of the active subcarrier indices in the VD of each subcarrier group and we use $\mathcal{I}_{S I}(\beta)\left(\beta=1,2, \ldots, N_{S I}\right)$ to denote all possible realizations of the active subcarrier indices. Additionally, there are $N_{q, l}=(Q L)^{K}$ realizations of $\mathbf{X}$, which represents $K$ STSK codewords in (8) and we apply $\mathcal{X}_{q, l}(\varphi)\left(\varphi=1,2, \ldots, N_{q, l}\right)$ to indicate all realizations of the $K$ STSK codewords in the proposed system model. Then the ML detector of each subcarrier group is formulated as:

$$
<\hat{\gamma}, \hat{\beta}, \hat{\varphi}>=\arg \min _{\gamma, \beta, \varphi}\left\|\mathbf{Y}-\mathbf{H} \overline{\mathcal{I}}_{A C}(\gamma) \overline{\mathbf{A}} \mathcal{I}_{S I}(\beta) \mathcal{X}_{q, l}(\varphi)\right\|^{2}
$$

where $\hat{\gamma}, \hat{\beta}$ and $\hat{\varphi}$ represent the estimates of the active TAs realization index, the active subcarriers realization index and the index of the realization for $K$ STSK codewords in each subcarrier group, respectively.

At the receiver, the ML detector carries out a full search for exploring all possible realizations, while having a complexity order of $\mathcal{O}\left[N_{A C} N_{S I}(Q L)^{K}\right]$ per subcarrier group. More precisely, the total computational complexity of the ML detector of (9) can be expressed as $\quad \mathcal{O}_{M L}\left[\left(N_{r} N_{t} M N_{f}^{3}+N_{r} M^{2} N_{f}^{2} N_{v}+N_{r} N_{f} M^{2} N_{v} K\right.\right.$ $\left.\left.+N_{r} N_{f} M K T\right) N_{A C} N_{S I}(Q L)^{K}\right]$, which may become excessive in practice for larger values of $Q$ and $L$, for a higher-dimensional VD, and/or for larger number of TAs. Therefore, a reduced-complexity detection algorithm will be proposed in Section III-A3.

2) Average BEP Analysis of ML Detection: For the sake of improved clarity, we rewrite the per-subcarrier-group received signal model of (8) as

$$
\mathbf{Y}=\mathbf{H} \overline{\mathbf{S}}+\mathbf{W}
$$

where $\overline{\mathbf{S}} \in \mathbb{C}^{N_{t} N_{f} \times T}$ is the equivalent data matrix which has $\left(N_{A C} N_{S I}(Q L)^{K}\right)$ possible realizations. Additionally, it is sufficient to determine the overall system performance by exploring the Pairwise Error (PE) events for a single subcarrier group, because the $\mathrm{PE}$ events of different subcarrier groups are the same [31]. If the matrix $\overline{\mathbf{S}}$ is transmitted and it is erroneously detected as $\widehat{\mathbf{S}}$, the receiver may make decision errors concerning either the active TAs, or the active SI and the STSK codewords per subcarrier group. Then the Conditional Pairwise Error Probability (CPEP) of the system model given in (10) is expressed as [31]

$$
P(\overline{\mathbf{S}} \rightarrow \widehat{\overline{\mathbf{S}}} \mid \mathbf{H})=Q\left(\sqrt{\frac{\|\mathbf{H}(\overline{\mathbf{S}}-\widehat{\overline{\mathbf{S}}})\|^{2}}{2 \sigma_{n}^{2}}}\right)
$$

Let $\mathbf{D}_{\overline{\mathbf{S}}}=\overline{\mathbf{S}}-\widehat{\overline{\mathbf{S}}}$ denote the difference matrix between $\overline{\mathbf{S}}$ and $\widehat{\widehat{S}}$. Applying $\mathbf{D}_{\overline{\mathbf{S}}}$ to (11), we have

$$
P(\overline{\mathbf{S}} \rightarrow \widehat{\overline{\mathbf{S}}} \mid \mathbf{H})=Q\left(\sqrt{\frac{\left\|\mathbf{H D}_{\overline{\mathbf{S}}}\right\|^{2}}{2 \sigma_{n}^{2}}}\right)=\frac{1}{\pi} \int_{0}^{\pi / 2} \exp (\xi \alpha) d \theta
$$

where we have $\xi=\left\|\mathbf{H D}_{\overline{\mathbf{S}}}\right\|^{2}=$ $\sum_{i=1}^{N_{f}} \sum_{r=1}^{N_{r}}\left(\mathbf{H}_{i}\right)_{r} \mathbf{D}_{\overline{\mathbf{S}}_{i}} \mathbf{D}_{\overline{\mathbf{S}}_{i}}^{H}\left(\mathbf{H}_{i}\right)_{r}^{H}, \quad \alpha=-\frac{1}{4 \sigma_{n}^{2} \sin ^{2} \theta}$ and $\left(\mathbf{H}_{i}\right)_{r}\left(i=1,2, \ldots, N_{f}\right.$ and $\left.r=1,2, \ldots, N_{r}\right)$ is used for denoting the $r$-th row of the $i$-th channel matrix given in (4). Then the computation of the Unconditional Pairwise Error 
Probability (UPEP) $P(\overline{\mathbf{S}} \rightarrow \widehat{\widehat{\mathbf{S}}})$ is obtained by taking the expectation relative to all possible channel realizations, while integrating the CPEP in (12) over the probability density function (pdf) of $\xi$ and yielding

$$
P(\overline{\mathbf{S}} \rightarrow \widehat{\overline{\mathbf{S}}})=\mathbb{E}_{\mathbf{H}}\{P(\overline{\mathbf{S}} \rightarrow \widehat{\widehat{\mathbf{S}}} \mid \mathbf{H})\}=\frac{1}{\pi} \int_{0}^{\pi / 2} \prod_{i=1}^{N_{f}} M_{\xi}(\alpha) d \theta,
$$

where $M_{\xi}(\alpha)$ represents the moment generating function of $\xi$ and we have

$$
\begin{aligned}
M_{\xi}(\alpha) & =\prod_{i=1}^{N_{f}}\left(\frac{1}{\operatorname{det}\left(\mathbf{I}_{N_{t}}-\alpha \mathbb{E}\left\{\left(\mathbf{H}_{i}\right)_{r}^{H}\left(\mathbf{H}_{i}\right)_{r}\right\} \mathbf{D}_{\overline{\mathbf{S}}_{i}} \mathbf{D}_{\overline{\mathbf{S}}_{i}}^{H}\right)}\right)^{N_{r}} \\
& =\prod_{i=1}^{N_{f}}\left(\frac{1}{1-\alpha\left\|\mathbf{D}_{\overline{\mathbf{S}}_{i}}\right\|^{2}}\right)^{N_{r}} .
\end{aligned}
$$

Upon substituting (15) into (13), the UPEP is finally obtained as

$$
P(\overline{\mathbf{S}} \rightarrow \widehat{\overline{\mathbf{S}}})=\frac{1}{\pi} \int_{0}^{\pi / 2} \prod_{i=1}^{N_{f}}\left(\frac{\sin ^{2} \theta}{\sin ^{2} \theta+\frac{\| \mathbf{D}_{\overline{\mathbf{S}}_{i} \|^{2}}}{4 \sigma_{n}^{2}}}\right)^{N_{r}} d \theta .
$$

After obtaining the UPEP in (16), an upper bound on the ABEP based on union bounding can be obtained by

$$
P_{B} \leq \frac{1}{b N(\overline{\mathbf{S}})} \sum_{\overline{\mathbf{S}}} \sum_{\widehat{\mathbf{S}}} P(\overline{\mathbf{S}} \rightarrow \widehat{\widehat{\mathbf{S}}}) d(\overline{\mathbf{S}}, \widehat{\overline{\mathbf{S}}}),
$$

where $b$ is the number of information bits carried by each subcarrier group, $N(\overline{\mathbf{S}})=N_{A C} N_{S I}(Q L)^{K}$ denotes the total number of possible realizations of $\overline{\mathbf{S}}$ per subcarrier group and $d(\overline{\mathbf{S}}, \widehat{\widehat{\mathbf{S}}})$ is used for representing the number of bit errors involved in the PE event of $(\overline{\mathbf{S}} \rightarrow \widehat{\mathbf{S}})$.

3) Reduced-Complexity Detection: In order to reduce the search-space of the ML detector of (9), in this section we propose a reduced-complexity detector. According to the received signal model $\mathbf{Y}$ of (8), there are three search items to be detected, namely the active TAs, the active subcarriers and the STSK codewords allocated to the active subcarriers, and for the sake of further analysis, we rewrite (8) as

$$
\begin{aligned}
\mathbf{Y} & =\mathbf{H} \overline{\mathbf{I}}_{A C} \overline{\mathbf{A}} \mathbf{I}_{S I} \mathbf{X}+\mathbf{W}=\mathbf{H} \overline{\mathbf{S}}+\mathbf{W} \\
& =\overline{\mathbf{H}} \mathbf{S}^{V D}+\mathbf{W} \\
& =\mathbf{\Phi} \mathbf{X}+\mathbf{W}
\end{aligned}
$$

where by definition we have $\overline{\mathbf{S}} \in \mathbb{C}^{N_{t} N_{f} \times T}=\overline{\mathbf{I}}_{A C} \mathbf{S}^{F D}$ for denoting the equivalent data matrix that has $N_{A C} \cdot N_{S I} \cdot(Q L)^{K}$ possible realizations, $\overline{\mathbf{H}} \in \mathbb{C}^{N_{r} N_{f} \times M N_{v}}=\mathbf{H} \overline{\mathbf{I}}_{A C} \overline{\mathbf{A}}$ is used for representing the equivalent channel matrix that has $N_{A C}$ possible realizations and $\Phi \in \mathbb{C}^{N_{r} N_{f} \times M K}$ for denoting the equivalent matrix which indicates having $N_{A C} \cdot N_{S I}$ possible joint support identifications, including the active TAs and active subcarriers. In terms of the sparsity characteristics of $\overline{\mathbf{S}}$ in (10) and $\mathbf{S}^{V D}$ in (18), the detector can be designed according to the CS principles [30].

Specifically, we use the matrix $\mathbf{Y} \in \mathbb{C}^{N_{r} N_{f} \times T}$ in (10) to explicitly represent the $N_{f}$ FD signals received from $N_{r}$ RAs over $T$ time slots and each column of $\mathbf{Y}$ can be regarded as a measurement vector according to the CS principles, where the joint support identification $\boldsymbol{\Phi}$ in our scenario is shared by $T$ measurement vectors from $\mathbf{Y}$, which is a manifestation of the MMV problem of CS principles [32]. Various recovery algorithms have been proposed for the MMV scenario, while in this work we propose a S-MP algorithm inspired by the classic Subspace Pursuit (SP) algorithm of [33] which is one of the popular greedy algorithms and it is used to solve the Single Measurement Vector (SMV) problem of [33].

The proposed S-MP procedure is described in Algorithm 1, where the $T$ measurement vectors of $\mathbf{Y}$ received are detected simultaneously in order to improve the performance.

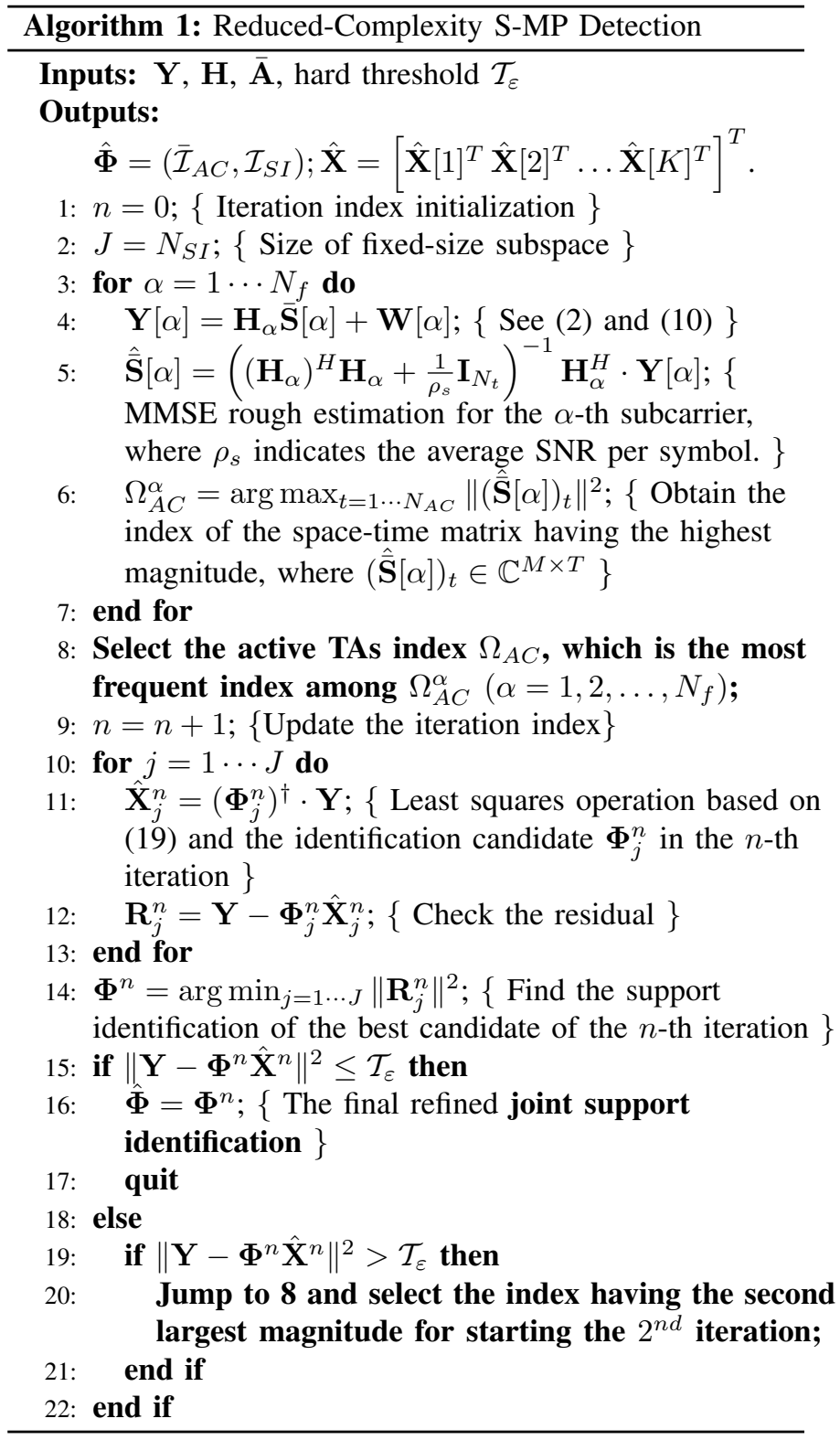

More specifically, the main differences between the proposed S-MP algorithm and the classical SP algorithm lie in two aspects: 1) The proposed S-MP algorithm is specificly designed for solving the MMV problem in our scenario and it 
is invoked for jointly detecting both the active TAs in the SD and the active subcarriers in the VD in our case as described in Algorithm 1, for achieving an improved performance. By contrast, the classical SP algorithm is only suitable for singledomain sparsity; 2) The most significant difference between the proposed S-MP and the classical SP algorithms lies in their specific approach invoked for generating the subspace during each iteration. In the classical SP algorithm, a fixedsize estimated subspace is maintained and refined during each iteration. On the other hand, in the proposed S-MP algorithm, during each iteration the algorithm exploits an $N_{S I}$-size subspace represented as $\boldsymbol{\Phi}_{j}^{n}\left(j=1,2, \ldots, N_{S I}\right)$, including the estimated active TA support identifications and the $N_{S I}$ possible active subcarrier support identifications, where the tested joint support identification resulting in the least residual errors is selected as the most reliable candidate during the iteration and then it is evaluated by comparing it to the hard threshold $\mathcal{T}_{\varepsilon}$ for terminating the iterations.

Based on the reduced-complexity S-MP procedure introcuded in Algorithm 1, the maximum number of iterations is $n=N_{A C}$, where $N_{A C} N_{S I}$ possible joint realizations will be explored and the complexity order per subcarrier group is given by $\mathcal{O}\left(N_{A C} N_{S I}+Q L K\right)$. Furthermore, most of the computational requirements of the proposed S-MP algorithm are imposed by the least squares operations carried out within the iterations. Therefore, the exact computational complexity of the proposed S-MP algorithm having $n=N_{A C}$ iterations is $\mathcal{O}_{S-M P}=$ $\left[\left(M K N_{r} N_{f}(2 M K+T)\right) N_{A C} N_{S I}+K Q L(M T)\right]$. Specifically, compared to the computational complexity of the ML detector of Section III-A1, the proposed S-MP algorithm significantly reduces the influence of having large $K, Q$, or $L$ values in the proposed system.

\section{B. Soft-Decision Detection}

Having introduced the hard-decision based ML detection and S-MP detection in Section III, we now introduce softdecision based detections. As illustrated in Fig. 6, we employ a two-stage concatenated CS-aided multi-dimensional IM system for achieving a near-capacity performance. Specifically, the channel encoder of Fig. 6 encodes the input bits by applying a RSC code for generating the coded bit sequence $c$ and the interleaver $\Pi$ is used for generating the interleaved stream $u$ to avoid the influence of bursty channel errors. Then the interleaved bit sequence $u$ of each subcarrier group is conveyed by the proposed CS-aided multi-dimensional IM modulator described in Section II-A. At the receiver of Fig. 6, the SISO decoder iteratively exchanges soft extrinsic information in the form of LLRs [34] with the RSC decoder. In Fig. 6, $L(\cdot)$ represents the LLRs of the bit sequence considered and the subscripts $i$ and $o$ illustrate the demodulator and RSC decoder, respectively, which are also termed as inner decoder and outer decoder. Additionally, the subscripts $a$ and $e$ indicate $a$ prior $i$ information and extrinsic information, respectively. To be specific, the demodulator's extrinsic output LLRs $L_{i, e}(u)$ are deinterleaved by a soft-bit deinterleaver to generate $L_{o, a}(c)$, which is the input of the outer RSC decoder as a priori

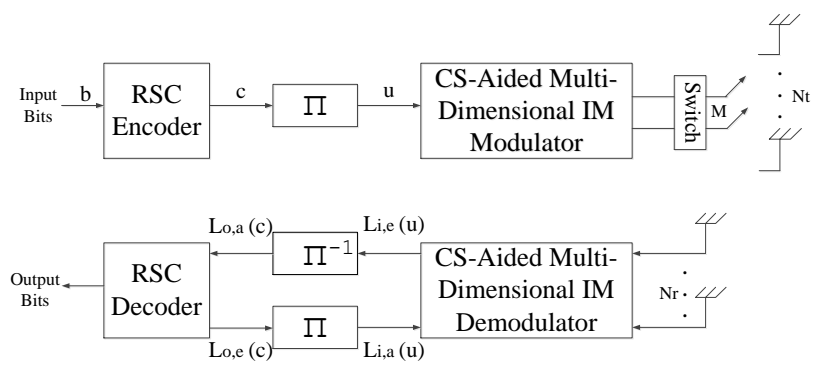

Fig. 6. The receiver architecture of the soft-decision assisted CS-aided multidimensional IM scheme.

LLRs. Then the extrinsic LLRs $L_{o, e}(c)$ of all the channelcoded bits of each subcarrier group are calculated by the Logarithmic Maximum a posteriori (Log-MAP) algorithm [27], [35], where the calculated extrinsic information $L_{o, e}(c)$ is then fed back to the demodulator as the a priori information $L_{i, a}(u)$ after the interleaver of Fig. 6 . Then the improved extrinsic LLRs are calculated by the demodulator and further Inner-Outer (IO) iterations are employed.

1) ML-based Soft-Decision Detection: According to the equivalent system model of (8) and the probabilistic representation of the STSK decoder invoked in [3], the conditional probability $p\left(\mathbf{Y} \mid \mathcal{X}_{\gamma, \beta, \varphi}\right)$ of receiving the group signal $\mathbf{Y}$ is given by

$$
\begin{aligned}
& p\left(\mathbf{Y} \mid \mathcal{X}_{\gamma, \beta, \varphi}\right) \\
& =\frac{1}{\left(\pi N_{0}\right)^{N T}} \exp \left(-\frac{\left\|\mathbf{Y}-\mathbf{H} \overline{\mathcal{I}}_{A C}(\gamma) \overline{\mathbf{A}} \mathcal{I}_{S I}(\beta) \mathcal{X}_{q, l}(\varphi)\right\|^{2}}{N_{0}}\right),
\end{aligned}
$$

where $\mathcal{X}_{\gamma, \beta, \varphi}$ represents STSK codewords at the $\beta$-th realization of active subcarriers, which are transmitted through the $\varphi$-th realization of active TAs, and $N_{0}$ is the noise power, where we have $\sigma_{n}^{2}=N_{0} / 2$ with $N_{0} / 2$ representing the double-sided noise power spectral density. The equivalent received signal $\mathbf{Y}$ per subcarrier group carries $B$ channelcoded bits $\mathbf{u}=\left[u_{1}, u_{2}, \ldots, u_{B}\right]$ and the extrinsic LLR of bit $u_{l}(l=1,2 \ldots, B)$ is expressed by (21) [28]. In (21), $\mathcal{X}_{1}^{l}$ and $\mathcal{X}_{0}^{l}$ represent a subset of the legitimate equivalent signal $\mathcal{X}$ corresponding to bit $u_{l}$ when $u_{l}=1$ and $u_{l}=0$, respectively, yielding $\mathcal{X}_{1}^{l} \equiv\left\{\mathcal{X}_{\gamma, \beta, \varphi} \in \mathcal{X}: u_{l}=1\right\}$ and $\mathcal{X}_{0}^{l} \equiv\left\{\mathcal{X}_{\gamma, \beta, \varphi} \in \mathcal{X}: u_{l}=0\right\}$. Additionally, $L_{a}(\cdot)$ in (21) represents the a priori information based on the feedback from the RSC decoder to the proposed modulator. Furthermore, the Approximate Log-MAP (Approx-Log-MAP) algorithm based on the Jacobian maximum operation [29] is implemented for the sake of simplifying the soft-decisions and the algorithm is given by [27], [36],

$$
L_{e}\left(u_{l}\right)=\operatorname{jac}_{\mathcal{X}_{\gamma, \beta, \varphi} \in \mathcal{X}_{1}^{l}}\left(\lambda_{\gamma, \beta, \varphi}\right)-\operatorname{jac}_{\mathcal{X}_{\gamma, \beta, \varphi} \in \mathcal{X}_{0}^{l}}\left(\lambda_{\gamma, \beta, \varphi}\right),
$$

where $\mathrm{jac}(\cdot)$ denotes the Jacobian maximum operation [29] and the intrinsic metric of $\lambda_{\gamma, \beta, \varphi}$ is expressed as

$$
\begin{aligned}
\lambda_{\gamma, \beta, \varphi}= & \left(-\left\|\mathbf{Y}-\mathbf{H} \overline{\mathcal{I}}_{A C}(\gamma) \overline{\mathbf{A}} \mathcal{I}_{S I}(\beta) \mathcal{X}_{q, l}(\varphi)\right\|^{2} / N_{0}\right. \\
& \left.+\sum_{j \neq l} u_{j} L_{a}\left(u_{j}\right)\right)
\end{aligned}
$$




$$
\begin{aligned}
L_{e}\left(u_{l}\right)= & \ln \frac{\sum_{\mathcal{X}_{\gamma, \beta, \varphi} \in \mathcal{X}_{1}^{l}} p\left(\mathbf{Y} \mid \mathcal{X}_{\gamma, \beta, \varphi}\right) \exp \left[\sum_{j \neq l} u_{j} L_{a}\left(u_{j}\right)\right]}{\sum_{\mathcal{X}_{\gamma, \beta, \varphi} \in \mathcal{X}_{0}^{l}} p\left(\mathbf{Y} \mid \mathcal{X}_{\gamma, \beta, \varphi}\right) \exp \left[\sum_{j \neq l} b_{j} L_{a}\left(b_{j}\right)\right]} \\
= & \ln \frac{\sum_{\mathcal{X}_{\gamma, \beta, \varphi} \in \mathcal{X}_{1}^{l}} \exp \left[-\left\|\mathbf{Y}-\mathbf{H} \overline{\mathcal{I}}_{A C}(\gamma) \overline{\mathbf{A}} \mathcal{I}_{S I}(\beta) \mathcal{X}_{q, l}(\varphi)\right\|^{2} / N_{0}+\sum_{j \neq l} u_{j} L_{a}\left(u_{j}\right)\right]}{\sum_{\mathcal{X}_{\gamma, \beta, \varphi} \in \mathcal{X}_{0}^{l}} \exp \left[-\left\|\mathbf{Y}-\mathbf{H} \overline{\mathcal{I}}_{A C}(\gamma) \overline{\mathbf{A}} \mathcal{I}_{S I}(\beta) \mathcal{X}_{q, l}(\varphi)\right\|^{2} / N_{0}+\sum_{j \neq l} u_{j} L_{a}\left(u_{j}\right)\right]} .
\end{aligned}
$$

2) S-MP-based Soft-Decision Detection: In order to reduce the computational complexity of the soft decoder, soft-decision based reduced-complexity S-MP detection is invoked. To be specific, the equivalent received signal $\mathbf{Y}$ per subcarrier group carries in total of $B$ channel-coded bits, where the first $B_{1}$ channel-coded bits $\mathbf{u}^{1}=\left[u_{1}^{1}, u_{2}^{1}, \ldots, u_{B_{1}}^{1}\right]$ are invoked for selecting the joint realizations of active TAs and active subcarriers, e.g. $B_{1}=\log \left(N_{A C}\right)+\log \left(N_{S I}\right)$, and the remaining $B_{2}$ channel-coded bits $\mathbf{u}^{2}=\left[u_{1}^{2}, u_{2}^{2}, \ldots, u_{B_{2}}^{2}\right]$ are used for encoding $K$ STSK codewords, where $B_{2}=K \log (Q L)$. Then at the first step of the S-MP-based soft-detection, the joint realizations of active TAs and active subcarriers are detected by applying our CS technique. Explicitly, based on the estimation of the joint identification support presented in Algorithm 1, the extrinsic LLR of bit $u_{l}^{1}\left(l=1,2, \ldots, B_{1}\right)$ expressed with the aid of the Jacobian maximum operation is given by [28], [37]

$$
L_{e}\left(u_{l}^{1}\right)=\operatorname{jac}_{\boldsymbol{\Phi}_{\gamma, \beta} \in \boldsymbol{\Phi}_{1}^{l}}\left(\lambda_{\gamma, \beta}\right)-\operatorname{jac}_{\boldsymbol{\Phi}_{\gamma, \beta} \in \boldsymbol{\Phi}_{0}^{l}}\left(\lambda_{\gamma, \beta}\right),
$$

where the intrinsic metric of $\lambda_{\gamma, \beta}$ is represented as

$$
\lambda_{\gamma, \beta}=\left(-\left\|\mathbf{Y}-\boldsymbol{\Phi}_{\gamma, \beta} \boldsymbol{\Phi}_{\gamma, \beta}^{\dagger} \mathbf{Y}\right\|^{2} / N_{0}+\sum_{j \neq l} u_{j} L_{a}\left(u_{j}\right)\right) .
$$

Then, first hard decisions can be made based on the LLRs of $L_{e}\left(u_{l}^{1}\right)$ for $B_{1}$ channel-coded bits to obtain the joint realization $\hat{\Phi}$ of the active TAs and active subcarriers. At the second step of the soft-decision based S-MP algorithm, the extrinsic LLR of bit $u_{l}^{2}\left(l=1,2, \ldots, B_{2}\right)$ is expressed by employing the Jacobian maximum operation as

$$
L_{e}\left(u_{l}^{2}\right)=\operatorname{jac}_{\mathcal{X}_{q, l} \in \mathcal{X}_{1}^{l}}\left(\lambda_{q, l, k}\right)-\operatorname{jac}_{\mathcal{X}_{q, l} \in \mathcal{X}_{0}^{l}}\left(\lambda_{q, l, k}\right),
$$

where the intrinsic metric of $\lambda_{q, l, k}$ is given by

$$
\lambda_{q, l, k}=\left(-\left\|\hat{\mathbf{X}}(k)-\mathcal{X}_{q, l}\right\|^{2} / N_{0}+\sum_{j \neq l} u_{j} L_{a}\left(u_{j}\right)\right),
$$

and the estimated STSK codewords $\hat{\mathbf{X}}(k)(k=1, \ldots, K)$ seen in (27) are obtained based on the result of the first step according to $\hat{\mathbf{X}}(k)=\left(\hat{\mathbf{\Phi}}^{\dagger} \mathbf{Y}\right)_{k}$.

\section{Performance Analysis}

In this section, the performance of the proposed CS-aided multi-dimensional IM system considered for transmission over frequency-selective fading channels is characterized by simulations. In all simulations, we assume perfect Channel State Information (CSI) knowledge at the receiver. The BER performances of the schemes are evaluated by Monte-Carlo simulations. Additionally, we focus our attention on OFDM having $N_{c}=128$ subcarriers in the FD, followed by a $\left(L_{c p}=16\right)$-length CP, and the COST2017-TU12 Rayleigh Fading channel model is applied.

\section{A. Hard-Decision Performances vs Computational Complexity Comparison}

In Fig. 7, we investigate the BER performances of the proposed CS-aided multi-dimensional IM system using both the ML detector in Section III-A1 and the proposed S-MP detector in Section III-A3, the CS-aided subcarrier IM system ${ }^{5}$ using the ML detector and the classical OFDM-STSK system applying the ML detector at the same transmission rate of $R_{t}=1.3333 \mathrm{bits} / \mathrm{s} / \mathrm{Hz}$. Furthermore, the theoretical bounds of (17) have been added to Fig. 7, where it is observed that the upper bound derived becomes very tight upon increasing the SNR values for the proposed CS-aided multi-dimensional IM system. According to the BER performances of these four schemes explored in Fig. 7, we conclude that an improved performance can be achieved, when the number of indexed transmission entities is increased. Specifically, for 1.3333 bits/s/Hz, the proposed CS-aided multi-dimensional system using the ML detector achieves about $4.15 \mathrm{~dB}$ and $8.75 \mathrm{~dB}$ better performances than the CS-aided subcarrier IM system and the classical OFDM-STSK system, respectively, at the BER value of $10^{-5}$. Additionally, the proposed CS-aided multidimensional IM system using the proposed S-MP detector has about $4.15 \mathrm{~dB}$ worse performance than its ML counterpart, but about $4.6 \mathrm{~dB}$ better performance than the classical OFDMSTSK system using ML detection, again at the BER value of $10^{-5}$. The performance of the proposed system applying the proposed S-MP detection is capable of approaching that of its ML counterpart upon increasing the SNR. Additionally, observe from Fig. 7 and Table II, the proposed S-MP detector is capable of reducing the computational complexity of the ML detector at the cost of a modest performance loss. More specifically, the proposed S-MP detector is capable of eliminating the influence of the varying $(Q L)$ values on the computational complexity compared with the ML detector. Hence, there is a clear SNR versus computational complexity tradeoff.

\section{B. EXIT Chart Analysis}

The exchange of extrinsic information in the proposed system of Fig. 6 can be visualised by plotting the EXIT characteristics of the inner demodulator and the outer RSC decoder [28] [38].

\footnotetext{
${ }^{5}$ The CS-aided subcarrier IM system is a special case of the proposed CSaided multi-dimensional IM scheme, while having $N_{t}=M$.
} 
TABLE II

COMPARISON BetweEn THE COMPUTATIONAL COMPLEXITIES (IN NUMBER OF OPERATIONS) PER SUBCARRIER GROUP OF THE ML DETECTOR AND THE PROPOSED S-MP DETECTOR

\begin{tabular}{|c|c|c|c|c|c|}
\hline \multirow[t]{2}{*}{$(Q L)$} & \multicolumn{2}{|c|}{$\begin{array}{l}\text { Complexities of CS-aided Multi-Dimensional IM } \\
\text { Schemes with } N_{v}=16, N_{f}=8, K=2, N_{t}=8 \text {, } \\
\text { STSK }(2,2,2, Q, L) \text { and varying }(Q L)\end{array}$} & \multirow[t]{2}{*}{$N_{t}$} & \multicolumn{2}{|c|}{$\begin{array}{l}\text { Complexities of CS-aided Multi-Dimensional } \\
\text { IM } \\
\text { Schemes with } N_{v}=16, N_{f}=8, K=2 \text {, } \\
\text { STSK }(2,2,2,2,2) \text { and varying } N_{t}\end{array}$} \\
\hline & ML (III-A1) & S-MP (III-A3) & & ML (III-A1) & S-MP (III-A3) \\
\hline 2 & $2.13 \times 10^{6}$ & $1.64 \times 10^{5}$ & 2 & $1.4 \times 10^{5}$ & $4.1 \times 10^{4}$ \\
\hline 4 & $8.52 \times 10^{6}$ & $1.64 \times 10^{5}$ & 4 & $5.41 \times 10^{5}$ & $8.2 \times 10^{4}$ \\
\hline 8 & $3.41 \times 10^{7}$ & $1.64 \times 10^{5}$ & 8 & $2.13 \times 10^{6}$ & $1.64 \times 10^{5}$ \\
\hline 16 & $1.36 \times 10^{8}$ & $1.64 \times 10^{5}$ & 16 & $8.45 \times 10^{6}$ & $3.28 \times 10^{5}$ \\
\hline
\end{tabular}

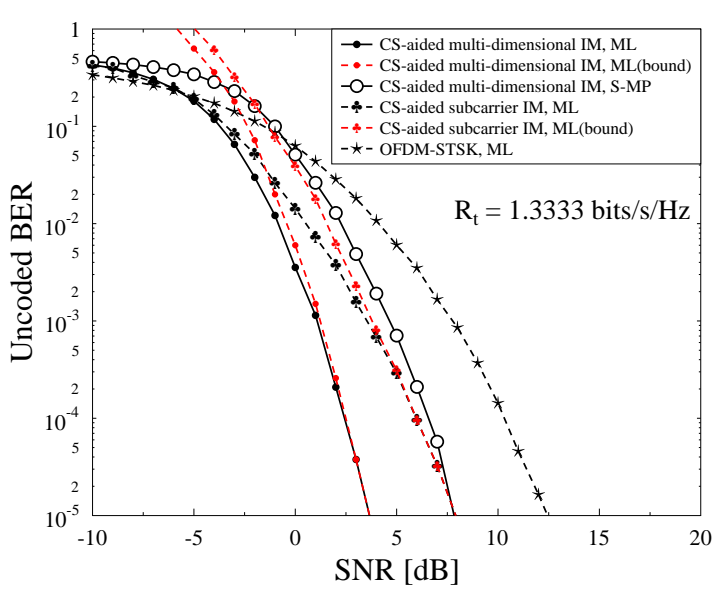

Fig. 7. BER performances of a) the proposed CS-aided multi-dimensional IM system using both ML detector and the proposed S-MP detector having $N_{v}=$ $16, N_{f}=8, K=2, N_{t}=8, \operatorname{STSK}(2,2,2,2,2)$, b) the CS-aided subcarrier IM system using ML detector having having $N_{v}=16, N_{f}=8, K=$ $2, N_{t}=2, \operatorname{STSK}(2,2,2,2,4)$ and c) the classical OFDM-STSK system using ML detector where 96 out of 128 subcarriers are used for transmitting $\operatorname{STSK}(2,2,2,2,2)$, at the same transmission rate of $R_{t}=1.3333 \mathrm{bits} / \mathrm{s} / \mathrm{Hz}$.

Fig. 8 shows the EXIT curves of the proposed CS-aided multi-dimensional IM scheme's inner demodulator for $E_{b} / N_{0}$ values spanning from $-7.75 \mathrm{~dB}$ to $-4.75 \mathrm{~dB}$ with a step size of $1.0 \mathrm{~dB}$, while using ML-based soft-detection. The system is characterised by $N_{f}=8, K=2, N_{v}=16$, $N_{t}=8$ and $\operatorname{STSK}(2,2,2,2,2)$, while employing a $1 / 2$-rate $\operatorname{RSC}(2,1,5)$ code relying on an interleaver depth of 192,000 bits. Additionally, Fig. 8 portrays the EXIT chart of the $1 / 2$ rate $\operatorname{RSC}(2,1,5)$ outer decoder. The Monte-Carlo simulationbased decoding trajectory is recorded at $E_{b} / N_{0}=-4.75$ $\mathrm{dB}$ between the inner demodulator and the outer decoder in Fig. 8, where the steps seen in the figure indicate the actual extrinsic mutual information transfer between them. According to the open-tunnel shown between the inner curve at $E_{b} / N_{0}=-4.75 \mathrm{~dB}$ and the outer curve, the iterative decoding process is expected to converge at around $E_{b} / N_{0}=-4.75 \mathrm{~dB}$ and at least 3 iterations are required for reaching the mutual information point $(1.0,1.0)$. Hence, the system is expected to produce an ifinitesimally low BER at about $E_{b} / N_{0}=-4.75$ $\mathrm{dB}$.

Fig. 9 shows the EXIT curves of the proposed CS-aided multi-dimensional IM system's inner demodulator for $E_{b} / N_{0}$

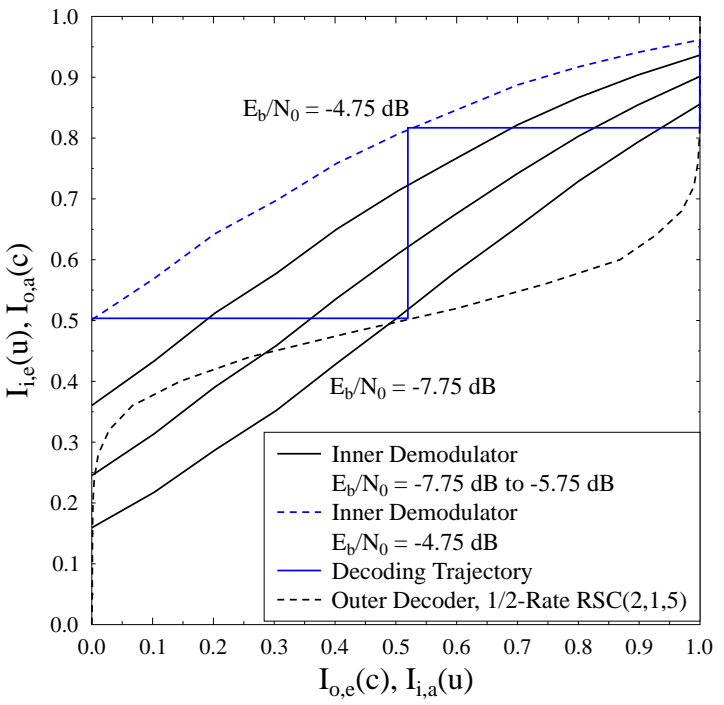

Fig. 8. The EXIT charts of the proposed CS-aided multi-dimensional IM system relying on the ML-based soft-detection, which is associated with the configuration: $N_{f}=8, K=2, N_{v}=16, N_{t}=8$ and $\operatorname{STSK}(2,2,2,2,2)$. A decoding trajectory is shown between the outer decoder's EXIT curve and the inner demodulator's EXIT curve at $E_{b} / N_{0}=-3.75 \mathrm{~dB}$, while using a $1 / 2$-rate $\operatorname{RSC}(2,1,5)$ with an interleaver depth of 192,000 bits.

values at $1.25 \mathrm{~dB}$ and at $2.25 \mathrm{~dB}$, while using S-MP-based soft-detection. Observe in Fig. 9 that the innner demodulator's EXIT curve shows a decreasing trend upon increasing $I_{i, a}(u)$. The reason for this behavior is that the $u$-length output data stream of the proposed CS-aided multi-dimensional IM demodulator using S-MP-based soft-detection is not Gaussian distributed and the LLRs of the $u$-length data stream denoted by $L_{i, e}(u)$ do not satisfy the consistency condition defined in [38] because of reducing the search space of the detector, although the calculation of $L_{i, e}(u)$ is based on the assumption that the $u$-length data stream is Gaussian distributed. A trivial solution to this problem is to try and find the probability distribution of the $u$-length output stream and compute $L_{i, e}(u)$ by using the actual probability density function (pdf). However, it is not straightforward to find a mathematical formula to model the pdf of the $u$-length data stream. Fortunately, however it is possible to compute the LLRs according to the histogram of the demodulated $u$-length data stream, computing 


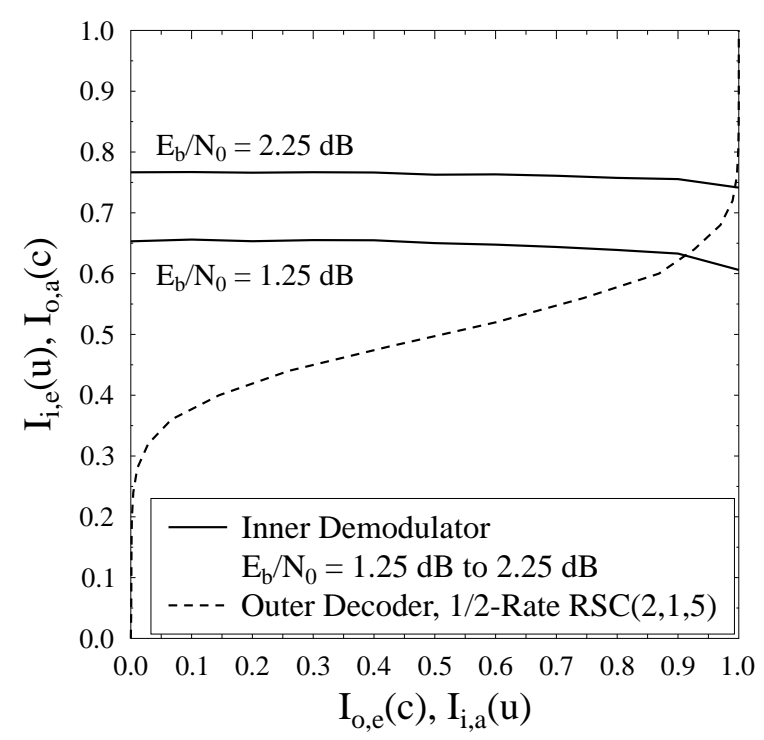

Fig. 9. The EXIT charts of the proposed CS-aided multi-dimensional IM system relying on the S-MP-based soft-detection without LLR postprocessing, which is associated with the configuration: $N_{f}=8, K=2, N_{v}=16$, $N_{t}=8$ and $\operatorname{STSK}(2,2,2,2,2)$. Additionally, the EXIT curve of the $1 / 2$ rate $\operatorname{RSC}(2,1,5)$ outer decoder with an interleaver depth of 192,000 bits is shown in the figure.

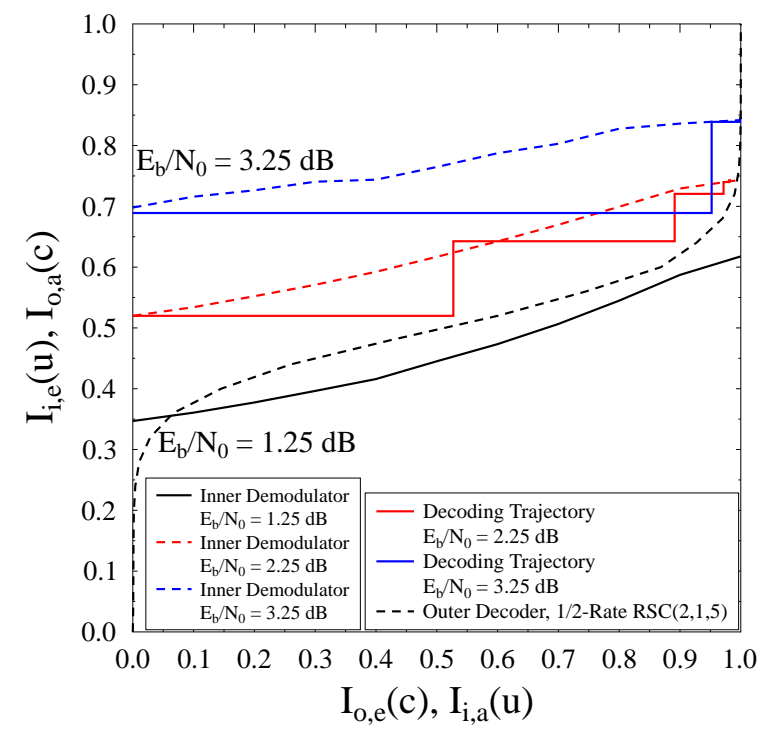

Fig. 10. The EXIT charts of the proposed CS-aided multi-dimensional IM system relying on the S-MP-based soft-detection with LLR postprocessing, which is associated with the configuration: $N_{f}=8, K=2, N_{v}=16$, $N_{t}=8$ and $\operatorname{STSK}(2,2,2,2,2)$. The decoding trajectories are shown between the outer decoder's EXIT curve and the inner demodulator's EXIT curves at $E_{b} / N_{0}=2.25 \mathrm{~dB}$ and $E_{b} / N_{0}=3.25 \mathrm{~dB}$, while using a $1 / 2$-rate $\operatorname{RSC}(2,1,5)$ with an interleaver depth of 192,000 bits.

the histogram for every received group is a complex and time- consuming process.

An empirical transformation of the $L_{i, e}(u)$ LLR values has been investigated for correcting the relationship between the LLRs and the corresponding probabilities to satisfy the consistency condition in [38], as proposed in [39]. This empirical transformation is referred to as LLR postprocessing and it can be expressed as

$$
L_{i, e}^{\dagger}(u)=\frac{L_{i, e}(u)}{\mathcal{A}},
$$

where $L_{i, e}^{\dagger}(u)$ represents the processed extrinsic LLRs passed from the inner demodulator to the deinterleaver of Fig. 6, which satisfies the consistency condition in [38], and $\mathcal{A}$ represents the empirical transformation coefficient, where we have $\mathcal{A}=8.0$ specifically for our scenario and it is obtained with the aid of simulations.

Fig. 10 shows the EXIT charts of the proposed CS-aided multi-dimensional IM system relying on the S-MP-based softdetection relying on the LLR postprocessing of (28), which is associated with the configuration: $N_{f}=8, K=2, N_{v}=16$, $N_{t}=8$ and $\operatorname{STSK}(2,2,2,2,2)$. The EXIT curves of the inner demapper using S-MP-based soft-detection recorded for the $E_{b} / N_{0}$ values spanning from $1.25 \mathrm{~dB}$ to $3.25 \mathrm{~dB}$ with a step size of $1.0 \mathrm{~dB}$ are shown in Fig. 10. As shown in Fig. 10, the inner demodulator's EXIT charts are shifted upwards upon increasing $E_{b} / N_{0}$ value. Additionally, the decoding trajectories at $E_{b} / N_{0}=2.25 \mathrm{~dB}$ and $E_{b} / N_{0}=3.25 \mathrm{~dB}$ between the inner demodulator and the $1 / 2$-rate $\operatorname{RSC}(2,1,5)$ outer decoder are recorded in Fig. 10, where the iterative decoding process is expected to converge around $E_{b} / N_{0}=3.25 \mathrm{~dB}$ and at least 3 iterations are required for approaching the mutual information point $(1.0,1.0)$.

\section{Capacity Analysis}

In this section, we formulate the DCMC capacity [40] of the proposed CS-aided multi-dimensional IM system using the full-search based ML detector presented in Section III-A1. Then the powerful tool of EXIT charts is utilised for determining the maximum achievable throughput of the proposed system using both the ML detector introduced in Section III-A1 and the S-MP detector presented in Section III-A3.

1) Discrete-Input Continuous-Output Memoryless Channel (DCMC) Capacity Analysis: The DCMC capacity of the proposed CS-aided multi-dimensional IM scheme is first developed for each subcarrier group, where $N_{A C}$ is the number of the active TAs combinations, $N_{S I}$ is that of the active subcarriers combinations and $K$ activated STSK codewords are included in each subcarrier group. Then the DCMC capacity per group developed is divided by $N_{f}$, namely by the number of FD subcarriers for determining the capacity per subcarrier. Based on the equivalent received signal model of (10), the DCMC capacity of the proposed scheme can be expressed as [40]

$$
\begin{aligned}
C^{D C M C}(S N R)= & \max _{\left.p\left(\overline{\mathbf{S}}^{i}\right)\right|_{i=1} ^{I}} \frac{1}{N_{f}} \sum_{i=1}^{I} \int p\left(\mathbf{Y} \mid \overline{\mathbf{S}}^{i}\right) p\left(\overline{\mathbf{S}}^{i}\right) \\
& \cdot \log _{2} \frac{p\left(\mathbf{Y} \mid \overline{\mathbf{S}}^{i}\right)}{\sum_{j=1}^{I} p\left(\mathbf{Y} \mid \overline{\mathbf{S}}^{j}\right) p\left(\overline{\mathbf{S}}^{j}\right)} d \mathbf{Y},
\end{aligned}
$$


where $I=N_{A C} N_{S I}(Q L)^{K}$ denotes the total number of realizations for each subcarrier group including the active TAs, active subcarriers and $K$ STSK codewords at the activated subcarriers. The DCMC capacity of (29) is maximized, when these realizations per subcarrier group are equiprobable, where we have $\left\{p\left(\overline{\mathbf{S}}^{i}\right)=\frac{1}{I}\right\}_{i=1}^{I}$. Furthermore, the conditional probability $p\left(\mathbf{Y} \mid \overline{\mathbf{S}}^{i}\right)$ of receiving $\mathbf{Y}$ given that the group-signal $\overline{\mathbf{S}}^{i}$ is transmitted over fading channels is determined by the probability density function of the noise, which is formulated as:

$$
p\left(\mathbf{Y} \mid \overline{\mathbf{S}}^{i}\right)=\frac{1}{\left(\pi N_{0}\right)^{N_{r} N_{f}}} \exp \left(-\frac{\left\|\mathbf{Y}-\mathbf{H} \overline{\mathbf{S}}^{i}\right\|^{2}}{N_{0}}\right)
$$

Based on (30), the DCMC capacity of (29) may be further simplified as

$$
\begin{aligned}
C^{D C M C}(S N R) & =\frac{1}{I N_{f}} \sum_{i=1}^{I} \mathbb{E}\left\{\log _{2}\left[\frac{I \cdot p\left(\mathbf{Y} \mid \overline{\mathbf{S}}^{i}\right)}{\sum_{j=1}^{I} p\left(\mathbf{Y} \mid \overline{\mathbf{S}}^{j}\right)}\right]\right\} \\
& =R-\frac{1}{I N_{f}} \mathbb{E}\left\{\log _{2}\left[\sum_{j=1}^{I} \exp \left(\Psi_{i, j}\right)\right]\right\},
\end{aligned}
$$

where we have $\Psi_{i, j}=\frac{-\left\|\mathbf{H}\left(\overline{\mathbf{S}}^{i}-\overline{\mathbf{S}}^{j}\right)+\mathbf{W}\right\|^{2}+\|\mathbf{W}\|^{2}}{N_{0}}$, while the system's throughput is given by $R=\frac{\log _{2} I}{N_{f}}$, which is expected to be achieved by the DCMC capacity of $C^{D C M C}(S N R)$ upon increasing the SNR values.

2) The Maximum Achievable Rate Using The EXIT Charts Tool: The EXIT charts can be utilised for determining the maximum achievable rate of the system at a given $E_{b} / N_{0}$ value [41]. Fig. 11 shows the maximum attainable rates of several proposed CS-aided multi-dimensional IM schemes associated with different configurations, while using both MLbased and S-MP-based detections. As shown in Fig. 11, the maximum achievable rate of the system tends to increase, when either the number of TAs $N_{t}$ or the number of ACs $N_{A C}$ increases. More specifically, it is shown in the figure that the maximum achievble rate of the system proposed in [22] and having $N_{t}=M$ is lower than that of the proposed CS-aided multi-dimensional IM system. The DCMC capacity curves evaluated based on (31) are portrayed in the figure as well. Observe in Fig. 11 that the maximum achievable rate of the proposed system evaluated from the EXIT chart closely matches the DCMC capacity curves. Additionally, it is shown in Fig. 11 that the maximum achievable rate curves of the proposed systems using S-MP detection is lower than that of ML detection due to the reduced search space. Furthermore, the channel encoding rate directly affects the achievable rate. The maximum rates of the systems employing a half-rate RSC decoder are shown in Fig. 11, where the systems have the following configurations: $\operatorname{STSK}(2,2,2,2,2), N_{f}=8, K=2$, $N_{v}=16$ and $N_{t}=8$. For visual clarify, the ML-based softdetection and S-MP-based soft-detection are marked in Fig. 11 by the red arrows at $E_{b} / N_{0}=-7.25 \mathrm{~dB}$ and $E_{b} / N_{0}=1.25$ $\mathrm{dB}$, respectively.

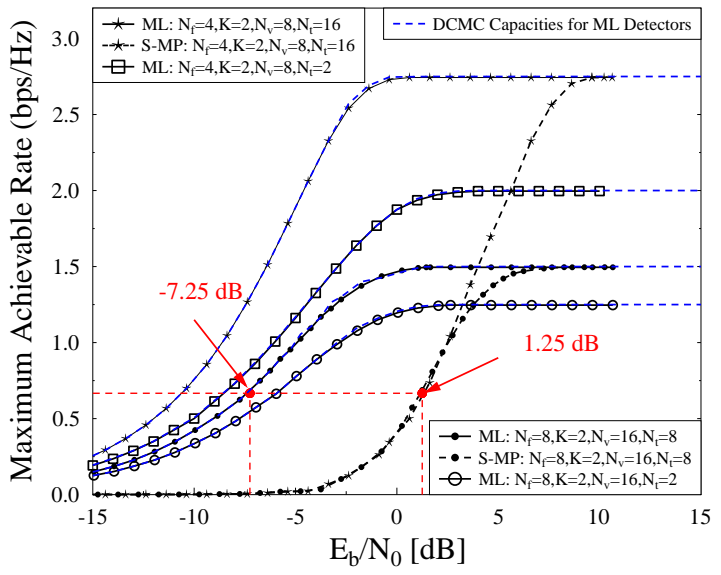

Fig. 11. Maximum achievable rates of several proposed CS-aided multidimensional IM schemes assiciated with different configurations, while using ML detection and S-MP detection. Additionally, the maximum rates of the proposed systems after employing a half-rate RSC decoder with configurations: $\operatorname{STSK}(2,2,2,2,2), N_{f}=8, K=2, N_{v}=16$ and $N_{t}=8$ ,using ML-based soft-detection and S-MP-based soft-detection are shown at $E_{b} / N_{0}=-7.25 \mathrm{~dB}$ and $E_{b} / N_{0}=1.25 \mathrm{~dB}$, respectively.

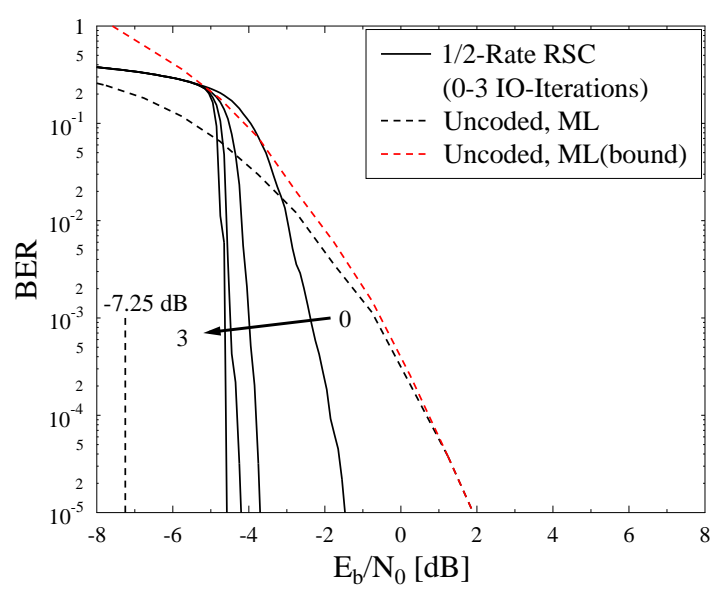

Fig. 12. BER performances of the 1/2-rate $\operatorname{RSC}(2,1,5)$-coded ML-based soft-detection system of Fig. 6 , while using 0 to 3 iterations.

\section{Coded BER Performance}

In this section, we show the coded BER performances of the proposed systems for the following configurations: $\operatorname{STSK}(2,2,2,2,2), N_{f}=8, K=2, N_{v}=16$ and $N_{t}=8$, while using ML-based soft-detection and S-MP-based softdetection, where the configurations used are the same as those in Fig. 8 and Fig. 10. A 1/2-rate $\operatorname{RSC}(2,1,5)$ encoder having an interleaver depth of 192,000 bits is employed in both systems. Fig. 12 shows the attainable BER performance of the $1 / 2$-rate $\operatorname{RSC}(2,1,5)$-coded system of Fig. 6 , while using an interleaver depth of 196,000 bits and 0 to 3 iterations. The BER performance of the uncoded system is also shown in the 


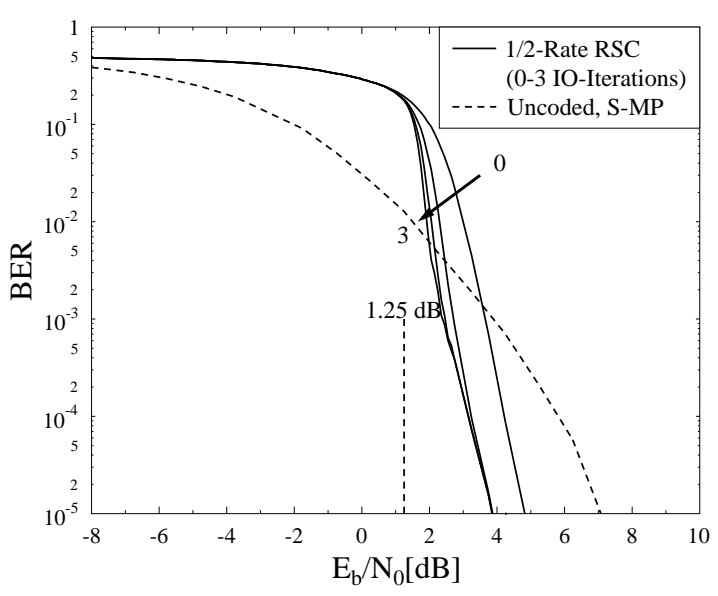

Fig. 13. BER performances of the 1/2-rate $\operatorname{RSC}(2,1,5)$-coded S-MP-based soft-detection system of Fig. 6, while using 0 to 3 iterations.

figure. Observe in Fig. 12 that there is an evident performance improvement, when increasing the number of iterations. As shown in Fig. 12, the system attains an infinitesimally low BER at around $E_{b} / N_{0}=-4.75 \mathrm{~dB}$, which matches the EXIT chart prediction seen in Fig. 8 and is within about $2.5 \mathrm{~dB}$ of the maximum achievable rate obtained using EXIT charts and shown in Fig. 11.

Fig. 13 shows the BER performances of the 1/2-rate $\operatorname{RSC}(2,1,5)$-coded CS-aided multi-dimensional IM system using the S-MP-based soft-detection of Fig. 6, while employing an interleaver depth of 196,000 bits and 0 to 3 iterations. The BER performance of the uncoded system is also shown in the figure. Note that the simulation results shown in Fig. 13 are obtained after the LLR postprocessing described in (28) of Section IV-B. Observe in Fig. 13 that there is an evident performance improvement, upon increasing the number of iterations. As shown in Fig 13, the system has no substantial performance gain beyond $E_{b} / N_{0}=3.25 \mathrm{~dB}$, which matches the EXIT chart prediction seen in Fig. 10, which is within about $2.0 \mathrm{~dB}$ from the maximum achievable rate obtained using EXIT charts and shown in Fig. 11.

\section{Design Guidelines}

In this paper, we proposed a novel CS-aided multidimentional IM scheme by exploring the TAs, subcarriers, time slots and dispersion matrices, which strikes flexible trade-offs among the attainable throughput, the detector's complexity and the achievable performance. Furthermore, the high design flexibility of the proposed scheme can be exploited by beneficially configuring the different components in the design, where the design flexibility makes the proposed scheme suitable for adaptive scenarios. Specifically, a CSbased reduced complexity detector, namely S-MP, was invoked for imposing a reduced computational complexity at a modest performance degradation, which makes the proposed multidimensional system eminently suitable for realistic scenarios, because the optimal ML detector is excessively complex. For example, let us consider a pair of system configurations: 1) $N_{v}=16, N_{f}=8, K=2, N_{t}=8, \operatorname{STSK}(2,2,2,8,2)$, 16 bits/group, $\mathcal{O}_{S-M P}=1.64 \times 10^{5}$ and 2) $N_{v}=16$, $N_{f}=8, K=2, N_{t}=2, \operatorname{STSK}(2,2,2,2,2), 10$ bits/group, $\mathcal{O}_{S-M P}=4.1 \times 10^{4}$, where System 1) has a higher throughput than System 2), and the complexity of System 1) is higher than that of System 2), but the performance of System 1) may be worse than that of System 2). Hence, the designer may strike the best trade-off between performance, throughput and complexity upon appropriately configuring the different components in the design.

\section{CONCLUSIONS}

In this paper, a novel CS-aided multi-dimensional IM scheme was proposed, where in addition to the TD and FD, the SD was also exploited for attaining additional diversity gain and attaining an improved performance. As observed in Fig. 7, at the same transmission rate, the proposed CS-aided multidimensional IM system employing the ML detector imposed a better performance compared both to the CS-aided subcarrier IM system of [22] and to the classical OFDM-STSK system. Then the reduced-complexity S-MP detector was proposed for providing a lower computational complexity with a modest BER degradation. Explicitly, observe from Fig. 7 and Table II, the proposed S-MP detector was capable of approaching the ML detector's performance while providing an affordable computational complexity. Furthermore, the CS-aided multidimensional IM SISO decoders were conceived in order to achieve a near-capacity performance. More specifically, EXIT charts were used not only for visually characterising the flow of soft-information between the constituent decoders of our concatenated structure, but also for evaluating the maximum achievable rate of the proposed system. Observe from Fig. 11, the proposed CS-aided multi-dimentional IM system imposed a higher maximum achievable rate compared to the CS-aided subcarrier IM system of [22].

\section{REFERENCES}

[1] E. Basar, "Index modulation techniques for $5 \mathrm{G}$ wireless networks," IEEE Communications Magazine, vol. 54, no. 7, pp. 168-175, July 2016.

[2] N. Ishikawa, S. Sugiura, and L. Hanzo, "Subcarrier-index modulation aided OFDM - will it work?" IEEE Access, vol. 4, pp. 2580-2593, May 2016.

[3] S. Sugiura, S. Chen, and L. Hanzo, "Coherent and differential spacetime shift keying: a dispersion matrix approach," IEEE Transactions on Communications, vol. 58, no. 11, pp. 3219-3230, November 2010.

[4] M. I. Kadir, S. Sugiura, S. Chen, and L. Hanzo, "Unified MIMOmulticarrier designs: a space-time shift keying approach," IEEE Communications Surveys Tutorials, vol. 17, no. 2, pp. 550-579, November 2015.

[5] R. Y. Mesleh, H. Haas, S. Sinanovic, C. W. Ahn, and S. Yun, "Spatial modulation," IEEE Transactions on Vehicular Technology, vol. 57, no. 4, pp. 2228-2241, July 2008.

[6] J. Jeganathan, A. Ghrayeb, L. Szczecinski, and A. Ceron, "Space shift keying modulation for MIMO channels," IEEE Transactions on Wireless Communications, vol. 8, no. 7, pp. 3692-3703, July 2009.

[7] M. Driusso, F. Babich, M. I. Kadir, and L. Hanzo, "OFDM aided space-time shift keying for dispersive downlink channels," in 2012 IEEE Vehicular Technology Conference (VTC Fall), Sept 2012, pp. 1-5.

[8] M. I. Kadir, S. Chen, K. Hari, K. Giridhar, and L. Hanzo, "OFDMaided differential space-time shift keying using iterative soft multiplesymbol differential sphere decoding," IEEE Transactions on Vehicular Technology, vol. 63, no. 8, pp. 4102-4108, Oct 2014. 
[9] I. A. Hemadeh, M. El-Hajjar, S. Won, and L. Hanzo, "Multi-set spacetime shift keying and space- frequency space-time shift keying for millimeter-wave communications," IEEE Access, vol. 5, pp. 8324-8342, December 2016.

[10] T. Datta, H. S. Eshwaraiah, and A. Chockalingam, "Generalized spaceand-frequency index modulation," IEEE Transactions on Vehicular Technology, vol. 65, no. 7, pp. 4911-4924, July 2016.

[11] E. Basar, U. Aygolu, E. Panayirci, and H. V. Poor, "Orthogonal frequency division multiplexing with index modulation," IEEE Transactions on Signal Processing, vol. 61, no. 22, pp. 5536-5549, Nov 2013.

[12] B. Shamasundar, S. Bhat, S. Jacob, and A. Chockalingam, "Multidimensional index modulation in wireless communications," IEEE Access, vol. 6, pp. 589-604, November 2018.

[13] R. Fan, Y. J. Yu, and Y. L. Guan, "Generalization of orthogonal frequency division multiplexing with index modulation," IEEE Transactions on Wireless Communications, vol. 14, no. 10, pp. 5350-5359, Oct 2015.

[14] M. Wen, E. Basar, Q. Li, B. Zheng, and M. Zhang, "Multiple-mode orthogonal frequency division multiplexing with index modulation," IEEE Transactions on Communications, vol. 65, no. 9, pp. 3892-3906, Sept 2017.

[15] E. Basar, "On multiple-input multiple-output OFDM with index modulation for next generation wireless networks," IEEE Transactions on Signal Processing, vol. 64, no. 15, pp. 3868-3878, Aug 2016.

[16] B. Zheng, M. Wen, E. Basar, and F. Chen, "Multiple-input multipleoutput OFDM with index modulation: low-complexity detector design," IEEE Transactions on Signal Processing, vol. 65, no. 11, pp. 2758-2772, June 2017.

[17] E. Basar and I. Altunbas, "Space-time channel modulation," IEEE Transactions on Vehicular Technology, vol. PP, no. 99, pp. 1-1, February 2017.

[18] D. L. Donoho, "Compressed sensing," IEEE Transactions on Information Theory, vol. 52, no. 4, pp. 1289-1306, April 2006.

[19] A. Garcia-Rodriguez and C. Masouros, "Low-complexity compressive sensing detection for spatial modulation in large-scale multiple access channels," IEEE Transactions on Communications, vol. 63, no. 7, pp. 2565-2579, July 2015.

[20] L. Xiao, P. Yang, Y. Xiao, S. Fan, M. D. Renzo, W. Xiang, and S. Li, "Efficient compressive sensing detectors for generalized spatial modulation systems," IEEE Transactions on Vehicular Technology, vol. 66, no. 2, pp. 1284-1298, Feb 2017.

[21] H. Zhang, L. L. Yang, and L. Hanzo, "Compressed sensing improves the performance of subcarrier index-modulation-assisted OFDM," IEEE Access, vol. 4, pp. 7859-7873, October 2016.

[22] S. Lu, I. A. Hemadeh, M. El-Hajjar, and L. Hanzo, "Compressed sensing-aided space-time frequency index modulation," IEEE Transactions on Vehicular Technology, pp. 1-1, 2018.

[23] C. E. Shannon, "A mathematical theory of communication," Bell Systems Technical Journal, vol. 27, pp. 623-656, October 1948.

[24] R. W. Hamming, "Error detecting and error correcting codes," Bell Systems Technical Journal, vol. 29, pp. 41-56, 1950.

[25] P. Elias, "Coding for noisy channels," IRE Conv. Rept., pp. 37-47, 1955.

[26] C. Berrou and A. Glavieux, "Near optimum error correcting coding and decoding: Turbo-codes," IEEE Trans. Commun., vol. 44, no. 10, pp. 1261-1271, October 1996.

[27] L. Hanzo, T. H. Liew, B. L. Yeap, R. Y. S. Tee, and S. X. Ng, Turbo coding, turbo equalisation and space-time coding:EXIT-chartaided near-capacity designs for wireless channels. Wiley-IEEE Press, 2011.

[28] S. ten Brink, "Convergence behavior of iteratively decoded parallel concatenated codes," IEEE Transactions on Communications, vol. 49, no. 10, pp. 1727-1737, Oct 2001.

[29] M. El-Hajjar and L. Hanzo, "EXIT charts for system design and analysis," IEEE Communications Surveys Tutorials, vol. 16, no. 1, pp. 127-153, May 2014.

[30] J. W. Choi, B. Shim, Y. Ding, B. Rao, and D. I. Kim, "Compressed sensing for wireless communications : useful tips and tricks," IEEE Communications Surveys Tutorials, vol. PP, no. 99, pp. 1-1, February 2017.

[31] L. Hanzo, T. H. Liew, and B. L. Yeap, SpaceTime block codes. IEEE, 2002. [Online]. Available: https://ieeexplore.ieee.org/xpl/articleDetails.jsp?arnumber $=5487950$

[32] J. D. Blanchard, M. Cermak, D. Hanle, and Y. Jing, "Greedy algorithms for joint sparse recovery," IEEE Transactions on Signal Processing, vol. 62, no. 7, pp. 1694-1704, April 2014.
[33] W. Dai and O. Milenkovic, "Subspace pursuit for compressive sensing signal reconstruction," IEEE Transactions on Information Theory, vol. 55, no. 5, pp. 2230-2249, May 2009.

[34] W. Koch and A. Baier, "Optimum and sub-optimum detection of coded data disturbed by time-varying intersymbol interference [applicable to digital mobile radio receivers]," in Global Telecommunications Conference, 1990, and Exhibition. 'Communications: Connecting the Future', GLOBECOM '90., IEEE, Dec 1990, pp. 1679-1684 vol.3.

[35] P. Robertson, E. Villebrun, and P. Hoeher, "A comparison of optimal and sub-optimal MAP decoding algorithms operating in the log domain," in Communications, 1995. ICC '95 Seattle, 'Gateway to Globalization', 1995 IEEE International Conference on, vol. 2, Jun 1995, pp. 10091013 vol.2.

[36] S. Benedetto and G. Montorsi, "Serial concatenation of block and convolutional codes," Electronics Letters, vol. 32, no. 10, pp. 887-888, May 1996.

[37] J. Hagenauer, E. Offer, and L. Papke, "Iterative decoding of binary block and convolutional codes," IEEE Transactions on Information Theory, vol. 42, no. 2, pp. 429-445, March 1996.

[38] J. Hagenauer, "The EXIT chart - introduction to extrinsic information transfer in iterative processing," in 2004 12th European Signal Processing Conference, Sept 2004, pp. 1541-1548.

[39] M. El-Hajjar, O. Alamri, R. G. Maunder, and L. Hanzo, "Layered steered space-time-spreading-aided generalized MC DS-CDMA," IEEE Transactions on Vehicular Technology, vol. 59, no. 2, pp. 999-1005, Feb 2010.

[40] C. Xu, S. Sugiura, S. X. Ng, P. Zhang, L. Wang, and L. Hanzo, "Two decades of MIMO design tradeoffs and reduced-complexity MIMO detection in near-capacity systems," IEEE Access, vol. 5, pp. 18564 $18632,2017$.

[41] A. Ashikhmin, G. Kramer, and S. ten Brink, "Extrinsic information transfer functions: model and erasure channel properties," IEEE Transactions on Information Theory, vol. 50, no. 11, pp. 2657-2673, Nov 2004.

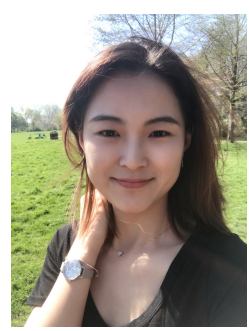

Siyao Lu received the B.Eng degree in electronic information science and technology from Dalian Maritime University of China and the M.Sc degree from the University of Southampton, U.K., where she is currently pursuing the Ph.D. degree with the Southampton Next Generation Wireless (NGW) Group under the supervision of Prof. L. Hanzo and Dr. M. El-Hajjar. Her research interests mainly include multifunctional MIMO, index modulation and millimeter-wave communications.

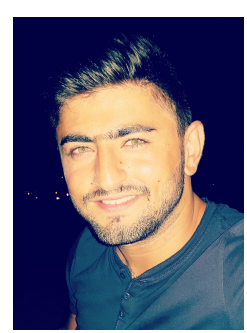

Ibrahim A. Hemadeh received the B.Eng. degree (with first class honor) in Computer and Communications Engineering from the Islamic University of Lebanon, Lebanon, in 2010. He then received the M.Sc. degree in Wireless Communications (with distinction) and the Ph.D. degree in Electronics and Electrical Engineering from the University of Southampton, UK, in 2012 and 2017, respectively. During 2017 he was a postdoctocal researcher at Southampton Next Generation Wireless (NGW) group, University of Southampton, UK and in March 2018 he moved to the University of Surrey. His research interests include millimeter-wave communications, multi-functional MIMO, multi-dimensional (time-space-and frequency) transceiver designs, channel coding as well as multi-user MIMO. 


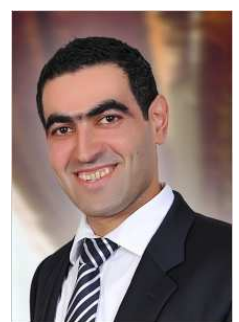

Mohammed El-Hajjar is an Associate Professor in the department of Electronics and Computer Science in the University of Southampton. He received his $\mathrm{PhD}$ in Wireless Communications from the University of Southampton, UK in 2008. Following the $\mathrm{PhD}$, he joined Imagination Technologies as a design engineer, where he worked on designing and developing Imaginations multi-standard communications platform, which resulted in three patents. He is the recipient of several academic awards and has published a Wiley-IEEE book and in excess of 80 journal and conference papers. Mohammeds research interests include the design 5of intelligent and energy-efficient transceivers, cross-layer optimization for large-scale networks, MIMO, millimeter wave communications and Radio over fiber network design.

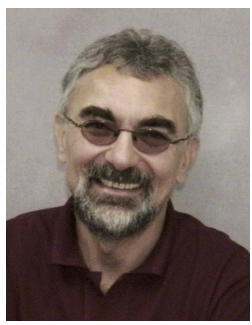

Lajos Hanzo (http://www-mobile.ecs.soton.ac.uk) FREng, F'04, FIET, Fellow of EURASIP, received his 5-year degree in electronics in 1976 and his doctorate in 1983 from the Technical University of Budapest. In 2009 he was awarded an honorary doctorate by the Technical University of Budapest and in 2015 by the University of Edinburgh. In 2016 he was admitted to the Hungarian Academy of Science. During his 40-year career in telecommunications he has held various research and academic posts in Hungary, Germany and the UK. Since 1986 he has been with the School of Electronics and Computer Science, University of Southampton, UK, where he holds the chair in telecommunications. He has successfully supervised $119 \mathrm{PhD}$ students, co-authored 18 John Wiley/IEEE Press books on mobile radio communications totalling in excess of 10000 pages, published $1800+$ research contributions at IEEE Xplore, acted both as TPC and General Chair of IEEE conferences, presented keynote lectures and has been awarded a number of distinctions. Currently he is directing a 60 -strong academic research team, working on a range of research projects in the field of wireless multimedia communications sponsored by industry, the Engineering and Physical Sciences Research Council (EPSRC) UK, the European Research Council's Advanced Fellow Grant and the Royal Society's Wolfson Research Merit Award. He is an enthusiastic supporter of industrial and academic liaison and he offers a range of industrial courses. He is also a Governor of the IEEE ComSoc and VTS. He is a former Editor-inChief of the IEEE Press and a former Chaired Professor also at Tsinghua University, Beijing. For further information on research in progress and associated publications please refer to http://www-mobile.ecs.soton.ac.uk 